NBER WORKING PAPER SERIES

\title{
WHEN TECHNOLOGICAL ADVANCE MEETS PHYSICIAN LEARNING IN DRUG
} PRESCRIBING

Domenico Depalo

Jay Bhattacharya

Vincenzo Atella

Federico Belotti

Working Paper 26202

http://www.nber.org/papers/w26202

\author{
NATIONAL BUREAU OF ECONOMIC RESEARCH \\ 1050 Massachusetts Avenue \\ Cambridge, MA 02138 \\ August 2019
}

We thank Jeremy D. Goldhaber-Fiebert, Salvador Navarro and seminar participants at IAAE in Montreal, Health Econometrics Workshop in Naples, Annual Health Econometrics Workshop in Baltimore. We would like to thank Dr Claudio Cricelli and SIMG for kindly providing the data included in the Health Search Database. Replication files and additional results will be available at the following webpage: http://sites.google.com/site/domdepalo/ Dr. Bhattacharya is grateful for support from the US National Institute on Aging (P30 AG17253) for his work on this paper. The views expressed herein are those of the authors and do not necessarily reflect the views or imply any responsibility for the National Bureau of Economic Research or their institutions.

NBER working papers are circulated for discussion and comment purposes. They have not been peer-reviewed or been subject to the review by the NBER Board of Directors that accompanies official NBER publications.

(C) 2019 by Domenico Depalo, Jay Bhattacharya, Vincenzo Atella, and Federico Belotti. All rights reserved. Short sections of text, not to exceed two paragraphs, may be quoted without explicit permission provided that full credit, including $\odot$ notice, is given to the source. 
When Technological Advance Meets Physician Learning in Drug Prescribing Domenico Depalo, Jay Bhattacharya, Vincenzo Atella, and Federico Belotti NBER Working Paper No. 26202

August 2019

JEL No. I1,I12,O33

\section{ABSTRACT}

The support for scientific investigation in biomedicine depends in part on the adoption of new knowledge into medical practice. We investigate how a technological advance, in the form of a large and influential 2010 randomized controlled study, changed physician practice in statin (a medication used to manage high cholesterol levels) prescribing. We analyze data representative of the Italian population for the period 2003-2014. Our analysis accounts for possible nonrandom sorting of patients into treatment. We show that both doctors and patients responded promptly to this technological shock, changing the mix of patients who received therapy, drug dosing, and frequency of testing for side effects, as well as patient adherence to therapy. The results show that investments in scientific knowledge can rapidly diffuse into practice in professions where continuing education is the norm.

Domenico Depalo

Bank of Italy

Via Nazionale 91

00184 Roma

Italy

DOMENICO.DEPALO@bancaditalia.it

Jay Bhattacharya

117 Encina Commons

CHP/PCOR

Stanford University

Stanford, CA 94305-6019

and NBER

jay@stanford.edu
Vincenzo Atella

University of Rome Tor Vergata

atella@uniroma2.it

Federico Belotti

Università degli Studi di Roma

"Tor Vergata"

Via Columbia

00133 Roma

Italy

belotti@economia.uniroma2.it 


\section{Introduction}

In every professional occupation (e.g. physicians, engineers, lawyers, accountants, etc.) new developments or events sometimes contradict previously held beliefs or norms. Such advancements could include the development of new technologies, the release of scientific studies, the promulgation of new regulations, or the passage of new laws, among countless other possibilities. Every occupation where such developments are common require continuing education efforts to maintain a good professional standing. Practitioners who fall behind, or who do not internalize the new information, risk practicing based on obsolete knowledge, harming their clients and the economy at large.

In this paper, we focus on the particularly important case of medical professionals. This case is important for at least two reasons: 1) if doctors do not keep up with the latest developments in their profession, they may harm their patients health and waste substantial sums of both public and private money; and 2) public support for health research is, in part, premised on the adoption of new discoveries into medical practice. We focus on a common but understudied form of technological advance - a randomized evaluation of an already existing product. This case is interesting because new information may influence the behavior of both physicians and patients. For physicians, important new technological developments should, in principle, lead to changes in optimal medical practice, especially when new evidence convincingly contradicts prior common medical wisdom. To the extent that patients are aware of this changing evidence, they may change their behavior (i.e. adherence to prescribed therapy) in response as well. The learning process may happen either because patients themselves become aware of the new evidence, or because changes in prescribed therapy induced by new information may improve clinical outcomes, or make side effects less likely or less severe. In short, important new technologies and new medical information should disrupt previous and current medical practice.

The primary aim of this study is to analyze the effects of a new technology in the form of an "information shock" that altered the scientific consensus on the side effect profile associated with statin medication, an important pharmaceutical product used to manage hypercholesterolemia (high serum cholesterol). We test how this new information diffused among physicians, and how this affected patient adherence to prescribed medication and health status.

Statins are among the most commonly prescribed medications around the world and a key tool that doctors use to prevent cardiovascular disease. However, statins have been historically seen to cause a potentially deadly side-effect - liver damage among a small fraction of the patients who take statins regularly. When 
statins were first introduced in the mid-1990s, standard practice (encoded in practice guidelines promulgated by professional societies) recommended conducting quarterly liver function tests (LFTs) for early evidence of liver damage. If the LFTs were found to be high, physicians reduced the statin dose, prescribed a different statin or even discontinued the drug. Some observational evidence in the mid-2000s suggested that this fear of liver damages was overstated, and some physicians started testing for liver side effects less frequently.

In late 2010, the GREACE randomized study confirmed that statins do not commonly cause mild liver damage, and in fact, improve liver function in a population of people who have liver disease from other causes. Since 2011, scientific societies modified guidelines to recommend that patients on statins do not need to have their liver enzyme levels routinely checked (Gillett and Norrell, 2011). Instead, guidelines suggest that liver function tests should be prescribed only when statin therapy on initiation or when the statin dose or type of statin prescribed is changed.

We take advantage of these discrete changes in information to measure how physician prescription and testing behaviors changed in response to changing evidence about the side effect profiles of statin medication in Italy. We also seek to understand how patient adherence to therapy changed in response to this changing evidence. To reach these goals, we first develop a simple theoretical model of technology adoption by physicians that allows us to derive testable implications and to conduct some comparative statics. We test the predictions of the model using observational data from a large longitudinal representative sample of Italian patients diagnosed with hypercholesterolemia between 2003 and 2014.

Given the history of the cholesterol treatment events, we focus on three distinct periods: 1) an early period (before 2006) when most physicians believed that statins might cause liver damage, and hence regular liver function testing was necessary; 2) a middle period (2006 - 2010 inclusive) where some observational studies suggested that statins might not be the cause of liver function abnormalities in patients taking statins; and 3) a later period (2010+) after the publication of the GREACE trial in 2010, which established that statins may actually help patients with liver function abnormalities. We discuss evidence that supports this division into three periods in the next section.

Our paper contributes to a growing literature that evaluates how scientific and technological advance alter physicians' practice (Currie and MacLeod, 2018, Avdic et al., 2018). In particular, Currie and MacLeod (2018) investigate the variations in physician decision making in the context of depression, a pathology where finding an optimal treatment involves experimentation with different drugs over time and where physician diagnostic skills are crucial. They conclude that the optimal management of health care should consist of a combination of constraints upon some choices, while allowing or even promoting discretion over a 
set of patient-specific treatments. Using data on percutaneous transluminal coronary angioplasty (PTCA) from Swedish hospitals, Avdic et al. (2018) investigate the physician adaptability to changes in treatment guidelines and the effects on patient outcomes, by exploiting unexpected information on the potentially adverse effects of using drug-eluting stents in cardiovascular diseases. They find substantial variation across providers in response to new information, with negative information generating more heterogeneous responses than positive information.

Unlike Currie and MacLeod (2018), the decision making in our paper is affected by an exogenous informational shock and by physician diagnostic skill. Unlike Avdic et al. (2018), who focus on stent implantation (a procedure that typically does not recur), we consider an asymptomatic (though potentially lethal) chronic condition managed over a prolonged period. In our case, the adherence of patients to recommended therapy (and hence clinical outcomes) depends upon the quality of the ongoing interaction between the doctor and the patient.

We organize the rest of the paper as follows. Section 2 discusses the changing evidence on statins; Section 3 presents our data set; Section 4 illustrates a simple model to guide our interpretation of our empirical results; Section 5 provides a description of our methods; Section 6 presents the empirical results; and finally, Section 7 offers concluding remarks.

\section{The changing evidence on statins}

Hypercholesterolemia is defined as a high level of cholesterol in the bloodstream. A high level of low-density lipoprotein (LDL) cholesterol is of medical concern because patients with high LDL levels are more likely to develop atherosclerosis, coronary artery disease, and associated heart diseases. Randomized trials have demonstrated that statin medications are a safe and effective way to reduce serum LDL levels and mortality risk. For instance, the 4S Study (Scandinavian Simvastatin Survival Study Group, 1994), among the earliest large statin randomized controlled trials (RCT), found that patients randomized to statins enjoyed a $35 \%$ reduction in LDL cholesterol and a 30\% reduction in mortality hazard relative to placebo-controlled patients.

However, like every drug, statin medications can cause adverse side effects. In the case of statins, an increase in blood levels of liver enzymes like alanine aminotransferase (ALT) - which in some cases indicate liver damage - was historically seen as the most common side effect. An early randomized study found fifteen times higher rates of elevated liver enzymes among patients assigned to high dose statins relative to placebo or low dose statins (Bradford et al. 1991). Physicians viewed this evidence of side effects as salient, 
and in studies of statin initiation they were found to be reluctant to prescribe statin therapy because of the risk of hepatotoxicity (Rzouq et al. 2010). Therefore, a finding of elevated LFTs might lead a physician to lower statin doses, change the prescribed statin molecule, or even recommend discontinuing statin therapy (Calderon et al., 2010). Given the concern about liver damage, until 2011 clinical guidelines suggested that physicians who prescribe statins for their patients should regularly test for changes in liver enzyme levels (McKenney et al., 2006, Gillett and Norrell, 2011), to help detect liver damage in patients on statins.

Evidence from around the world raised the idea that the link between statins, elevated liver enzyme levels, and permanent liver damage was not well established. Indeed in the early days of statin medications there were observational studies that found that elevated liver function tests did not necessarily indicate permanent liver damage and that a finding of elevated liver enzymes was often readily reversible (Mölgaard et al. 1991). Some further observational evidence published in the mid-2000s suggested that this fear of liver damage was probably overstated. A 2004 literature review concluded that routine liver function tests were often not ordered and that most cases of elevated liver enzymes were simply transitory (Sniderman, 2004). A series of observational studies of statin use among patients with pre-existing liver disease found the drug to be safe and effective, with no evidence of hepatotoxicity (Khorashadi et al. 2006, Segarra-Newnham et al. 2007; Lewis et al., 2007; Bhardwaj and Chalasani, 2007). A 2006 Italian study reviewed the evidence that statins cause liver damage, and concluded that, though the authors found some evidence for a relationship, there was not enough to support a causal link (Conforti et al. 2006). A 2006 American expert panel of hepatologists concluded that statin use was largely safe in terms of liver side effects (Cohen et al. 2006). However, given the evidence from the early statin RCTs the evidence was still mixed, the medical community continued to recommend testing to check for this type of side effects (Bays, 2006, Clarke and Mills, 2006).

This belief changed when, in late 2010, the GREACE study of statin medication use in a large population of Greek patients with abnormal liver tests was published in Lancet, a leading medical journal Athyros et al. 2010). The study showed that despite elevated liver enzyme levels, patients randomized to statin medications experienced lower rates of elevated liver enzymes relative to control patients, with a better cardiovascular response. This study was striking because its results were so unexpected given what most physicians believed about the effects of statins. If patients with liver disease show improvements in liver function after statin therapy, then the prior conventional wisdom that statins can cause low-grade liver damage in patients without liver disease is most likely incorrect. This, in turn, means that a finding of elevated liver function tests in the context of statin therapy is not evidence that the statin medication is leading to liver damage. The GREACE results suggested that statin medications could be continued despite elevated liver enzymes, and 
hence that patients on statins need not be tested regularly with LFTs (Pastori et al., 2015) 1

Ultimately, this reasoning led experts to reverse the recommendations in previous guidelines. Between 2011 and 2014, multiple American practice guidelines were modified to recommend that patients on statins did not need to have their liver enzyme levels checked regularly. They suggest instead that liver function tests are needed on initiation or when the statin dose or type of statin prescribed is changed (Gillett and Norrell, 2011; Bays et al. 2014). Though the Italian guideline for ordering liver function tests in the context of statin therapy has not changed, the GREACE study was widely known among Italian physicians, because pharmaceutical companies put considerable effort into spreading this information among primary care physicians through their commercial representatives ${ }^{2}$

\section{$3 \quad$ Data and Summary Statistics}

In this section, we describe the dataset, the selection criteria for our sample, our measures of drug potency and patient adherence to therapy and, finally, we present the sample summary statistics for all the variables we use in this study.

\subsection{The Italian Health Search Database}

Our data are drawn from the Health Search Database (HSD), a longitudinal observational database collected by the Italian College of General Practitioners (SIMG) since 1998. The HSD contains patient-level data from computer-based patient records reported by General Practitioners (GPs) throughout Italy. Every individual who is under the management of a participating GP is included in the HSD database even if he/she has no doctor visits in any given year.

Although GP participation is voluntary, GPs in the HSD are representative of the National Health Service (NHS) regional organization. PThe HSD tracks patients in proportion to the size of the Italian adult population within each region (Fabiani et al. 2004$) !^{3}$

Crucially for our analysis, in Italy, patients are matched to physicians via a largely exogenous process. The choice of physician by a patient is primarily influenced by the geographical location of patients and

\footnotetext{
${ }^{1}$ The GRAECE study did not contradict a previous finding that in some rare cases, statins can cause rhabdomyolysis, a serious condition involving muscle degradation that can lead to extensive liver and kidney damage. This rare side effect cannot typically be prevented or detected early with regular liver function tests.

${ }^{2}$ Personal communication with Dr. Claudio Cortese, lipidologist at the University of Rome Tor Vergata.

${ }^{3}$ Data have been collected routinely since 2000. More details on the extent to which the database represents the adult Italian population can be found at https://www.healthsearch.it/(Official website of the HSD project). Data from HSD have been used in several publications, both in clinical and social sciences peer-reviewed journals (see Mazzaglia et al., 2009 Atella and Kopinska 2014 Atella and Conti, 2014 Atella and D'Amico 2015 Atella et al., 2017, 2019, among others).
} 
physicians. Physicians are not allowed to reject a patient for service based on the patient's medical condition severity, nor they can select patients when they first register. Furthermore, there is no financial incentive that might induce physicians to select healthier or sicker patients.

Patient data are linked through a unique anonymous identifier to drug prescriptions, clinical events and diagnoses, hospital admissions, and causes of death. The information available includes the prescription dispensing date and drug characteristics (Anatomical Therapeutic Chemical or ATC code, which indicates the quantity and type of active ingredient and number of pills). Other observable characteristics are discussed in Section 3.3. GPs have routinely collected the data for the HSD since 2003, and our sample includes all data through 2014, the latest date available to us.

We selected patients based on two main inclusion criteria: 1) patients who receive a diagnosis of "pure hyper-cholesterolemia" (or familial hypercholesterolemia) and received at least one dose of statin between 2003 to 2014; and 2) patients born between 1925 and 1975, inclusive. We thus have an unbalanced panel of twelve years, with 11 years of follow-up for the cohort of patients who were observed to be on statins in 2003 and two years of follow-up for the 2012 cohort (that is, 2013 and 2014).

We analyze the data at the patient-quarter level. This choice represents a compromise aimed at reducing the number of observations with zero values in drug consumption, while permitting frequent changes in treatment and outcomes. The final sample consists of 103,737 patients (57,244 women and 46,493 men) for a total of $1,792,595$ observations.

We do not exclude patients who initiated statin therapy before 2003. First, the half-life of statins in humans depends on the molecule, but for all the statins we consider, it is less than 20 hours (Plakogiannis and Cohen, 2007) 4 Since the half-life of statin medications are so short, the effect of the drug on cholesterol levels for a patient who initiates therapy before 2003 will depend primarily on patient adherence during 2003 and not on drug-taking behavior before then. Second, including people who are already on therapy in 2003 may lead us to underestimate the effect of statin medications on cholesterol levels. Patients who routinely took statin medications before the start of our observation window in 2003 are likely to have already reached their statin-influenced equilibrium cholesterol level at the start of our observation window. Continuing to take the statin medications after 2003 is unlikely to lead to further lowering for patients already in physiological equilibrium. The results we present in this pa[er, are thus a conservative estimate of the effect of statin therapy on treatment outcomes.

\footnotetext{
${ }^{4}$ Half-life here indicates the amount of time it takes for half of the initial dose to be excreted from the body or metabolized into an inactive form.
} 


\subsection{Measuring Drug Potency and Patient Adherence}

We characterize the prescribed treatment regime using a univariate measure called the 'equipotency score.' This score accounts for the heterogeneous effect of different statin molecules in blocking cholesterol synthesis in the liver, and for the fact that higher doses of the same molecule will have a more powerful cholesterol synthesis blocking effect than lower doses Maron et al. (2000). Table 1 shows this conversion formula for the three statin molecules that we analyze in this study (simvastatin, atorvastatin, and rosuvastatin) $5^{5}$ We choose these three molecules because they were the most prescribed by Italian physicians.

The effect of the statin also depends upon the patient's adherence with the prescribed treatment regimen; a drug cannot affect if a patient decides not to take it ${ }^{6}$ We measure patient adherence by the Mean Possession Ratio (MPR) (Cramer et al., 2008, Atella et al., 2017). The MPR is the fraction of days over a quarter covered by all prescriptions for a drug:

$$
M P R=\left(\frac{\text { Sum of days' supply for all fills in quarter }}{\text { Number of days in quarter }}\right)
$$

The idea is that a patient who is poorly adherent to therapy does not need a refill prescription, and will consequently have a low MPR. The numerator of MPR is derived from the exact day when a refill occurs. However, patients need not wait until their statin supply is finished to order a refill; hence there might be some "overlap." To avoid an adherence indicator greater than 100\%, whenever a patient has some days of medications left from a previous refill, we add these extra days of medication to the next period, unless the covered days of the next refill are enough to be $100 \%$ adherent.

\subsection{Other Observable characteristics}

The Health Search Database includes detailed information on patients clinical histories. We calculate the Charlson Index, which is a standard summary measure of the number and severity of a patients health conditions, and a good predictor of a patients 10-year mortality risk (Charlson et al., 1987). The index takes on higher values for older patients and for each health condition a patient has. For example, a healthy

\footnotetext{
${ }^{5}$ According to this table, going from $20 \mathrm{mg}$ to $40 \mathrm{mg}$ of Simvastatin increases the expected LDL cholesterol reduction by one equipotency unit. Similarly, switching from $20 \mathrm{mg}$ of simvastatin to $20 \mathrm{mg}$ of atorvastatin or $10 \mathrm{mg}$ of rosuvastatin leads to an increase in the equipotency score because rosuvastatin is more biologically active than atorvastatin, and atorvastatin is more biologically active than simvastatin. Switching from $40 \mathrm{mg}$ of simvastatin to $20 \mathrm{mg}$ of atorvastatin or $5 \mathrm{mg}$ of rosuvastatin maintains the same equipotency level.

${ }^{6}$ According to the International Society for Pharmacoeconomics and Outcomes Research (ISPOR), medication compliance is commonly measured by persistent adherence to prescribed medical treatment. Adherence refers to the proportion of prescribed doses taken in the prescribed time interval, while persistence refers to the continued use of a prescribed therapy over time (Hughes et al. 2001).
} 
patient with no comorbidities has a Charlson score of zero, a patient with a history of acute myocardial infarction has a score of one, and a patient with a history of AIDS has a score of six. We aggregate scores above 4 in a unique category to avoid cells with a small sample size.

In addition to the Charlson Index, for each patient, we have available time-varying indicators of many important conditions including diabetes, hypertension, congestive heart failure, atrial fibrillation, vascular diseases, a history of cardiac bypass surgery or percutaneous coronary intervention (PCI), ischemic heart disease, or other cardiac conditions. In terms of diagnostic tests, we observe the number of times each patient is prescribed liver function tests by each physician, including alkaline phosphatase (ALP), aspartate aminotransferase (AST), alanine aminotransferase (ALT), albumin and bilirubin. Finally, we have a set of patient socio-demographic indicators, including age, sex, and the geographical region of residence.

\subsection{Summary Statistics}

Table 2 reports the list of variables we use in this paper, alongside descriptive statistics by sex. The table, in particular, reports the average yearly reduction in LDL-cholesterol, which is measured in SI units (mmol per liter) rather than in mg per $\mathrm{dL}$ (which are the standard units typically reported in the US) ${ }^{7}$ Using yearly change in LDL levels purges the statistic of variation due to seasonal effects (e.g., Christmas holiday diets may produce a high LDL-cholesterol level). In sensitivity checks, we define the change in LDL cholesterol at a quarterly level, with no qualitative change in our main results. As we can see in the table, females represent $55.0 \%$ of the sample, and the mean age is around 69 years for women and 65 for men. In accordance with the distribution of the Italian population, the majority of the patients are concentrated in the north of Italy (25.0\% North-west, $20.0 \%$ North-east), with the center accounting for about $18.0 \%$, and the south and the islands (Sardinia and Sicily) accounting for another $25 \%$.

The initial level of cholesterol (that is, the first observed cholesterol level for a patient in our sample) is higher for women $(120 \mathrm{mmol} / \mathrm{L})$ than for men $(110 \mathrm{mmol} / \mathrm{L})$. Men and women reduce their LDL cholesterol levels in a similar amount, by an average of $3.8 \%$ per year. The reduction of cholesterol is achieved thanks to statins, with an equipotency strength index (equip.) in the range of 4.5-4.6. The adherence rate (MPR) is high and larger for men (70\%) than for women $(65 \%)$.

The Charlson index is similar by gender (about 1.0). In about one-third of the sample, diabetes is present, and in about two thirds hypertension is present. Tests for liver dysfunction (aspartate aminotransferase and alanine aminotransferase levels in the blood) are prescribed about once per year (or 0.25 per quarter) on

\footnotetext{
${ }^{7}$ The reader can convert from $\mathrm{mmol} / \mathrm{L}$ to $\mathrm{mg} / \mathrm{d} \mathrm{L}$ by multiplying by 38.67 .
} 
average.

Table 3 reports the number of prescriptions recorded between 2003 and 2014 in our dataset by sex, dosage, and active ingredient. These numbers reflect specific physician prescribing patterns for simvastatin (Simva), atorvastatin (Ator), and rosuvastatin (Rosa) in Italy. The most prescribed active ingredients/dose combinations are Simvastatin 40mg, Atorvastatin 20mg, and Rosuvastatin 10mg.

\section{Theoretical model}

In this section, we propose a stylized model of physicians forming their beliefs about the efficacy and side effects of cholesterol medication, their optimal recommended choice of therapy given those beliefs and, finally, patients deciding about their adherence behavior in response to those recommendations. Our main aim is to generate simple comparative static predictions about the effects of an information shock on physician beliefs and patient outcomes. We use this model to guide the interpretation of our empirical analysis in later sections of the paper.

\subsection{Physician Objectives}

We model physician beliefs about the effects of statin medication along two dimensions - the effect of statins on the cholesterol level $(C)$ of a patient, and the occurrence of side effects $(S E)$ produced by the medication (such as liver or muscle damage). To simplify the notation and the algebra of our theoretical model, but without loss of generality, we impose that physicians take into account only the equipotency dose $(Q)$ in forming their prescription recommendation so that the model does not distinguish between switching to a more potent molecule and switching to a higher dose of the same molecule. We also assume that $S E$ can be measured by a single positive real-valued number, where larger values indicate either more or worse side effects for the patient (or both). For notational convenience, we leave implicit that all the beliefs and objective functions we consider are conditioned on observable characteristics $(X)$.

Higher serum cholesterol levels and more side effects are bad outcomes that physicians and patients want to minimize. To fit our discussion into the usual maximization framework, we introduce the following notation: $\bar{C}=-C$ and $\overline{S E}=-S E$ and, whenever it will improve the clarity of the presentation, will refer to it rather than to $C$ and $S E$.

Our focus in most of this discussion is on the decisions of doctors to recommend therapy. Therefore, we start with the physician utility function, with physicians serving as perfect agents for patients, which is 
common in this setting (Scott and Vick, 1999; Gafni et al., 1998). In Section 4.6 we allow patient behavior to deviate from physician recommendations. Physicians evaluate a combination of cholesterol and side effects outcomes with a von-Neumann-Morgenstern utility function $u(\bar{C}, \overline{S E} \mid Q)$, which is a nondecreasing function of both arguments. As a consequence, physician utility does not decrease when a patient's cholesterol or side effect levels decline:

$$
\frac{\partial u}{\partial \bar{C}} \geq 0 \text { and } \frac{\partial u}{\partial \overline{S E}} \geq 0
$$

\subsection{Physician Beliefs}

From a physician's (and patient's) point of view, prescribing (and taking) statin medications does not produce a certain outcome; rather, it produces a range of possible outcomes which are uncertain at the time of prescription. This uncertainty is a key feature of decision making by doctors, and so decisions will be based on physician beliefs about the probability of cholesterol control and side effects, for a given equipotency dose $Q: F(c, s e \mid Q)=P(\bar{C}<c, \overline{S E}<s e \mid Q)$. These beliefs, $F(\cdot)$, incorporate the scientific evidence regarding the clinical effects of statins as well as all other relevant information available to the doctor, such as patient clinical characteristics $(X)$. In particular, $F(\cdot)$ can be interpreted as incorporating physician beliefs about patient adherence to recommended therapy. We explore this issue further in Section 4.6 .

Based on the randomized clinical trial evidence we cite in Section 2, we assume that physicians believe that a higher equipotency dose will increase the probability of side effects and reduce the probability of a high cholesterol level (for a given side effect level). We formalize this assumption as:

$$
\begin{array}{r}
F(\overline{S E} \mid Q) \leq F\left(\overline{S E} \mid Q^{\prime}\right) \quad \forall Q^{\prime} \geq Q \\
F(\bar{C} \mid \overline{S E}, Q) \geq F\left(\bar{C} \mid \overline{S E}, Q^{\prime}\right) \quad \forall Q^{\prime} \geq Q .
\end{array}
$$

Equations (3) and (4) imply that, in expectation, an increase in the equipotency dose $Q$ reduces cholesterol levels and increase side effects. Applying our notational convention, we have $\forall Q^{\prime} \geq Q$ :

$$
\begin{array}{r}
E[\overline{S E} \mid Q] \geq E\left[\overline{S E} \mid Q^{\prime}\right] \\
E[\bar{C} \mid \overline{S E}, Q] \leq E\left[\bar{C} \mid \overline{S E}, Q^{\prime}\right] .
\end{array}
$$


In what follows, we will mostly rely on equations( 5 and (6), which we view as mild restrictions (as they make a statement only about the first moment of the $(C, S E)$ distribution) that are based on RCT evidence. Equations( 5) and (6) generate testable implications regarding the effect of statins on serum cholesterol levels.

\subsection{Changing Evidence and Changing Beliefs}

The next step consists in modeling how the release of new information could change physician beliefs. The evidence produced by the GREACE trial (primarily) revised the scientific view about the effect of statins on liver function damage, rather than the effect of statins on cholesterol. The latter effect was well established based on prior studies conducted in the 1990s and earlier, and the relation was accepted unanimously over time. The belief shock we are interested in, then, is the change in the probability of a side effect, for any given quantity $Q$. By definition of joint cumulative distribution function applied to the von-Neumann-Morgenstern utility function, for any belief, $F(\cdot)$ (hereafter $F$ for simplicity), we have:

$$
F(\bar{C}, \overline{S E} \mid Q)=F(\bar{C} \mid \overline{S E}, Q) F(\overline{S E} \mid Q)
$$

Here, for a given dose $\mathrm{Q}, F(\bar{C} \mid \overline{S E}, Q)$ is the conditional c.d.f. implied by $F$ on serum cholesterol for given side effects $\overline{S E}$, and $F(\overline{S E} \mid Q)$ is the marginal c.d.f. of side effects implied by $F$

Let $F^{P R E}$ and $F^{P O S T}$ represent beliefs about the relationship between equipotency dose, cholesterol levels, and side effect profiles that a well-informed physician will hold before and after the release of the GREACE trial. The fact that the GREACE trial did not change beliefs about the effect of statins on serum cholesterol levels can be formalized as:

$$
F^{P O S T}(\bar{C} \mid \overline{S E}, Q)=F^{P R E}(\bar{C} \mid \overline{S E}, Q)
$$

On the contrary, as the main findings of GREACE were focused on the hepatic side effects of statins, and in particular that statins are less likely to cause hepatic side effects than was previously believed, we have:

$$
F^{P O S T}(\overline{S E} \mid Q) \leq F^{P R E}(\overline{S E} \mid Q)
$$

which implies a first-order stochastic dominance relationship ( $F^{P O S T}$ dominates $\left.F^{P R E}\right)$. By combining the

\footnotetext{
${ }^{8}$ There is a little abuse of terminology, because both $F(\overline{S E} \mid \bar{C}, Q)$ and $F(\bar{C} \mid Q)$ are conditional c.d.f.s. However, we think that our terminology helps our exposition, while being innocuous for the rest of the paper.
} 
results in equation (8) and (9), from equation (7) it follows:

$$
F^{P O S T}(\bar{C}, \overline{S E} \mid Q) \leq F^{P R E}(\bar{C}, \overline{S E} \mid Q)
$$

Equation 9 implies that physician beliefs before the GREACE study is such that a given dose of statins would produce more side effects than a physician whose beliefs are based on the post-GREACE consensus:

$$
E^{P R E}[\overline{S E} \mid Q] \leq E^{P O S T}[\overline{S E} \mid Q]
$$

which should, in turn, be reflected in different patterns of diagnostic test prescriptions.

For this paper, we assume that GREACE unveiled the true relationship between LDL cholesterol levels, side effects and quantity of drugs 9

$$
F^{P O S T}=F^{0},
$$

where $F^{0}$ is the true relationship between $Q, C$ and $S E 10$

\footnotetext{
${ }^{9}$ The natural question that arises in this context is whether the results of the GREACE trial reflect the true relationship between statin dose, cholesterol levels, and side effects. Given the results of the GREACE trial, it should be clear that the pre-existing literature did not reflect reality, or else the results of the GREACE trial would have been quite different. In principle, though, the GREACE study is not necessarily definitive, and future studies could further refine scientific knowledge about these relationships.

${ }^{10} \mathrm{We}$ believe it is reasonable to assume $F^{P O S T}=F^{0}$ because, based on the current scientific consensus, the GREACE trial represents a closer approximation to $F^{0}$ than did beliefs engendered by the evidence base prior to GREACE $\left(F^{P R E}\right)$. We recognize that equation 12 is a strong assumption that simplifies our theoretical analysis considerably. This assumption could be wrong in two distinct ways. First, it is possible that in truth, there are fewer side effects from statin use than were measured in the GREACE trial, so that:

$$
F^{P R E}>F^{P O S T}>F^{0} \text {. }
$$

In this case, the results of our theoretical analysis still hold with minimal modification, and the empirical results we report regarding the effect of practice size on physician practice are conservative (that is, the expectation based on GREACE may be an underestimate of the true effect). Alternatively, it is possible that future studies will conclude that the GREACE study was incorrectly optimistic about the side effect profile of statins. In that case, either

$$
F^{P R E}>F^{0}>F^{P O S T}
$$

or even,

$$
F^{0}>F^{P R E}>F^{P O S T} \text {. }
$$

In the former case, the qualitative results of the model will still be valid, although the quantitative results would be overly optimistic. In the latter, the interpretation of our result comparing doctors who treat many patients against doctors who treat fewer patients would need to be modified. If a future study were to find that the GREACE was incorrect and that statins do cause hepatic side effects, we would then be forced to conclude that physicians with large practices are currently behaving (and have been behaving even before GREACE) based on false beliefs about the side effect profiles of statins. They have learned nothing from their more extensive clinical practice and are not updating their beliefs in response to the evidence in front of them.
} 


\subsection{Physician Learning}

So far, we have modeled the effect of a large one-time shock in beliefs generated by the GREACE trial. In fact, physicians always update their beliefs based on their private knowledge. There are various channels to acquire private knowledge. In our case, we model this aspect as a process of learning by doing, where the more patients a physician treats for hypercholesterolemia, the higher is the knowledge the physician accumulates. It then follows that the more patients a physician treats, the closer that physician beliefs will be to reality. We model the physician learning process as a function of the number of patients treated using a Bayesian framework.

Let $N$ be the number of patients that a physician has treated for this condition and let $g(N)$ be a function with the following properties 11

$$
\begin{aligned}
g(0) & =0 \\
\lim _{N \rightarrow \infty} g(N) & =1 \\
g^{\prime}(N) & \geq 0 .
\end{aligned}
$$

We model physician beliefs, $F^{d o c}$, as follows:

$$
F^{d o c}(\overline{S E} \mid \bar{C}, Q)=g(N) F^{0}(\overline{S E} \mid \bar{C}, Q)+(1-g(N)) F^{P R E}(\overline{S E} \mid \bar{C}, Q) .
$$

As the number of patients increases $(N \rightarrow \infty)$, physician beliefs come closer to the true effects of prescribing statins. If physicians could observe the entire population, their belief would coincide with $F^{0}$ (by our assumptions about $g(N)$ ). As this is not possible, we should expect an effect on the prescription behavior of all physicians.

However, even before the GREACE trial was published physicians who treated a larger number of patients should have behaved as if statins did not produce liver side effects: for those physicians, the publication of the GREACE trial should have had a smaller effect in changing their practice. By contrast, the GREACE study should have sharply changed the beliefs of physicians who were treating fewer patients (and thus had

\footnotetext{
${ }^{11}$ In principle, doctors update their beliefs based on the outcomes experienced by their patients that adhere to the therapy recommended by the doctor. If a doctor has many patients, most of whom ignore physician advice, the doctor will not learn very much. The reader should think of $N$ as the number of patients seen by the doctor who comply with doctor recommendations. Doctors who are better at convincing their patients to adhere to therapy will then have a larger $N$, despite seeing fewer patients than doctors who do a poor job communicating the importance of adherence.
} 
beliefs closer to $F^{P R E}$ ); for these physicians, we should observe sharper decreases in rates of liver function testing after GREACE than in larger practices.

\subsection{Optimal Prescribing}

We assume that doctors' utility follows the standard von-Neumann-Morgenstern assumptions, with the doctor taking the expectation given their own belief, $F^{d o c}$, about the efficacy and side effects of statins. Doctors choose $Q$ in a way that solves:

$$
\max _{Q} \quad U=E^{d o c}[u(\bar{C}, \overline{S E})]
$$

where the expectation is taken relative to the doctor's beliefs (Equation 13).

The optimal $Q_{d o c}^{*}$ is chosen to maximize $U$ with expectations set based on $F^{d o c}$, which we assume is fixed near the optimum. The first order condition is given by:

$$
\frac{d U}{d Q}=\frac{\partial u}{\partial E^{d o c}[\bar{C}]} \frac{\partial E^{d o c}[\bar{C}]}{\partial Q}+\frac{\partial u}{\partial E^{d o c}[\overline{S E}]} \frac{\partial E^{d o c}[\overline{S E}]}{\partial Q}=0
$$

which, in the expectations of the GP, balances the marginal decrease in cholesterol level from a higher statin equipotency dose against the marginal increase in side effects.

While this procedure identifies the optimal dose given the doctor's expectations about the safety and efficacy of statin medication, if the doctor's beliefs do not match the truth $\left(F^{d o c} \neq F^{0}\right)$, then the observed cholesterol levels and side effect patterns will differ from what the doctor expects. In particular, the doctor expects to find side effect levels equal to $E^{d o c}\left[\overline{S E} \mid Q_{d o c}^{*}\right]$ in response to prescribing the optimal dose, but in fact observes $E^{0}\left[\overline{S E} \mid Q_{d o c}^{*}\right]$, where the latter expectation is taken with respect to $F^{0}$. Since $F^{0}=F^{P O S T} \leq F^{P R E}$ (see equations 9 and 10), we have:

$$
E^{0}\left[\overline{S E} \mid Q_{d o c}^{*}\right] \geq E^{d o c}\left[\overline{S E} \mid Q_{d o c}^{*}\right]
$$

In our empirical analysis, we indirectly test this implication by analyzing the frequency at which doctors order a test for liver damage. The rationale behind our approach is that as physician beliefs come closer to $F^{0}$, we expect them to order fewer liver function tests since, as GREACE demonstrated, statins are unlikely to have such side effects.

Likewise, as doctor's beliefs tend toward $F^{0}$ - either because GREACE has been released or a doctor sees 
a large number of patients -, $F^{P O S T} \leq F^{P R E}$ so that the expected cost in terms of side effects decreases and therefore the optimal dose will increase, which implies:

$$
Q_{P R E}^{*}<Q_{P O S T}^{*}
$$

Since the optimal equipotency dose is predicted to be higher in the post-GREACE period (and for doctors whose beliefs are closer to $F^{0}$ ), we also predict lower cholesterol levels in the treated population relative to the pre-GREACE period.

This theoretical framework implies changes in physician prescribing on both the intensive and extensive margins, on an individual patient basis. That is, the GREACE shock should have increased both the optimal dose for patients already on a statin (intensive margin), and expanded the set of patients who optimally should be on statin therapy (extensive margin).

A testable implication for the intensive margin is simple. If our theory is correct, we should observe a rightward shift in the equipotency dose distribution of statin prescriptions over time, since post-GREACE physicians are less likely to think that a high dose will lead to liver harm.

A testable implication on the extensive margin for our theory is that sicker patients are selected for high dose statin therapy in the post-GREACE period than were selected during the pre-GREACE period. The reasoning is analogous to the intensive margin case. If our theory is right, prior to 2006 and prior to GREACE, the perceived risk of hepatic side effects limited physicians' willingness to try statins on their high risk patients. If the side effects were to occur, sicker would be in worse position to recover from them than healthier patients. After the information shock, the perceived risk of hepatic side effects diminished, which in turn made physicians more comfortable prescribing statins to their sicker patients.

\subsection{Patient Adherence}

Up to now, our modeling effort has emphasized the role that doctors play in prescribing therapy. Of course, patients play a crucial role in therapeutic decision making as well. If a patient decides against taking a medication, it does not matter how wise or well-informed the physician's prescription recommendation was, the therapy will not affect the patient's outcomes. So, to correctly model the relationship between statin dose and the response of cholesterol levels, it is crucial to consider how the recommended drug/dose combination, the potential side effects, and patient adherence interact with each other.

Lack of adherence is particularly salient when considering treatment for a condition like hypercholes- 
terolemia. As we discussed in Section 2, high serum cholesterol is an asymptomatic condition, and outside of a health care setting where serum cholesterol levels are measured, patients may never see any tangible or visible benefit from adhering to the prescribed therapy. By contrast, though rare, some of the side effects that are caused by statin medication - such as muscle pain- may be painful and easily observable. Patients may also incorrectly attribute other symptoms they are experiencing to a prescribed statin, even if the medical literature does not support such a relationship. In situations like this, adherence to prescribed statin medications is likely to be low.

Lack of adherence arises because patients have their own preferences and beliefs that physicians only imperfectly reflect. Though physicians in principle have more knowledge about the evidence regarding the effects of medications, patients certainly have their own ideas. Along these lines, patient adherence to therapy depends on how much patients trust the recommendations of their doctor. If doctors do a poor job in communicating with their patients, they will be less likely to adhere to the doctors' recommendations. Of course, some doctors are better than others in communicating with their patients (Fichera et al., 2018, Depalo, 2018). It shall be clear that this evidence has important empirical implications in terms of our identification strategy that we discuss at length in Section 5.2

A higher recommended dose by a doctor who is good at eliciting adherence will, according to our theory, reduce a patient's cholesterol level. Information shocks like GREACE can intensify the incentives that physicians have to increase the dose or push to increased adherence. We examine these issues directly in the empirical section of the paper.

\subsection{Testable Aspects of the Model}

In this section, we list the testable implications of our model, along with two testable assumptions on which the model is built. We intend this summary to guide the presentation of our empirical results in the rest of the paper.

We start with the list of necessary conditions (NC), which must hold for our model:

NC1 Increased adherence to prescribed statin therapy reduces serum cholesterol (see Section 4.2);

NC2 Increases in the prescribed equipotency dose of statins $(Q)$ reduces serum cholesterol levels (across different periods; see equations (4) and (6)).

Provided these conditions are satisfied, our model generates the following testable implications. As physician beliefs come closer to the true effect of statins $\left(F^{0}\right)$ : 
TI1 Physicians who treat more patients with statins (even in the pre-GREACE period) are likely to have beliefs closer to $F^{0}$ than physicians who treat fewer patients (Section 4.4);

TI2 Physicians will be willing to prescribe statins to sicker patients since the side effects of statins are less likely to occur or less serious than the physicians believed under $F^{P R E}$ (see discussion extensive margins in Section 4.5;

TI3 After 2010, the number of liver tests prescribed by physicians has decreased relative to the preGREACE period (see equation 16 in Section 4.5;

TI4 When new information arrives, physicians will be willing to prescribe higher equipotency doses holding fixed patient disease severity (see eq 17 in Section 4.5.

In addition to these testable implications on the physician side, our model allows us to make predictions on patient outcomes:

TI5 GREACE will increase the adherence to medication (see Section 4.6). Since the optimal equipotency dose is predicted to be higher in the post-GREACE period, the model predicts lower cholesterol levels in the treated population in the post-GREACE period relative to the pre-GREACE period (see Section 4.6.

\section{$5 \quad$ Empirical Methods}

In this section, we present our econometric approach to testing the hypotheses arising from our theoretical model presented in Section 4. In Section 5.1, we show that the change in the information set available to doctors about the side effect profiles of statins over time is exogenous and, therefore, can be used to identify causal treatment effects.

In Section 5.2, we discuss our approach to addressing the possibility that health status differences across patients and treatment are due to unobservable characteristics of the patients. In particular, we use physician propensity to prescribe statins for other patients in the physician's practice. Our argument for this

instrumenting strategy relies on the process by which patients and physicians match with each other in the Italian healthcare system. 


\subsection{Identification of Changing Cholesterol Management Patterns}

Our model implies that physicians learn either through the dissemination of new scientific results or by treating more patients. If the GREACE trial altered the scientific consensus about the side-effect patterns of statin medications, then the clinical characteristics of the treated population should have changed in the post-GREACE era (with sicker patients under treatment), as should the relationship between diagnostic testing and drug switching behavior. Our model also predicts that physicians who had a high patient volume in the pre-GREACE period should have been less affected in their prescribing behavior by the release of GREACE because their beliefs were already closer to the truth.

To test these predictions, we divide our analysis into three periods (before 2006, 2006-2010 inclusive, and $2011+$ ). During the early period, the conventional wisdom was that statins might cause liver damage in some cases. In the middle period, there was some non-randomized evidence that the effect of statins on liver function might not be directly causal. While this evidence was available before 2006, we chose this threshold as it coincides with a publication on the topic specific to Italy (Conforti et al., 2006). Finally, in the late period after the publication of the GREACE randomized trial, statins were presumed not to cause liver dysfunction. Therefore, we conduct all our analyses separately for those three periods.

The fact that this information shock could not be anticipated and is thus exogenous (see Section 1) is a primary argument for the identification of separate effects in the three periods. We permit the possibility that some physicians may have anticipated the results of the GREACE trial by exploring the behavior of physicians in high volume practices.

Our conditioning set includes the Charlson index (a composite comorbidity score explained in Section 3), age, sex, region of residence, and indicators for the presence of chronic diseases like diabetes, hypertension, congestive heart failure, atrial fibrillation, vascular diseases, a history of cardiac bypass surgery or percutaneous coronary intervention (PCI), ischemic heart disease, or other cardiac conditions. The use of such an extensive set of covariates in these regressions reduces the chance of bias due to observable differences between patients assigned to different statin molecule/dose combinations, though we relax the assumption of no unobserved differences by employing instrumental variable methods.

\subsection{Physician Habits and Persuasiveness as Instrumental Variables}

In an observational study such as this, there is always the possibility of unobserved health differences between patients on different treatment. Health status may depend on a variety of clinical and economic factors known 
to both patients and physicians, but not to us. To address this possibility, we employ the physician-specific statin adherence rates (MPR) and physician-specific statin equipotency switches as instrumental variables (IV). These choices of IVs are consistent with our theoretical model (see Section 4.6) and justified by the idea that physician experience using the drug on other patients may lead them to change their beliefs about its efficacy and side effect profile, which in turn will influence both the recommended prescription dose and how effective physicians are in encouraging their patients to adhere to the drug.

The physician-level adherence variable is a measure of the effectiveness of a physician's communication and persuasion abilities. The economic argument for this instrument is thus that a physician's communication skills should correlate strongly with a patient's own adherence to the physician's recommended therapy, but should have no direct effect on medical outcomes such as serum LDL level except through adherence. For a given patient, we calculate this instrument by calculating the average MPR over all the other statin patients seen by the physician. We use this IV in models where the endogenous right-hand-side variable is related to a patient's adherence to therapy.

The physician-level dosing variable is a measure, in part, of a physician's belief about the safety of prescribing high dose statins. One implication of our model is that, holding fixed a patient's health status, a physician whose prior is that statins are not likely to cause liver side-effects will be less likey to switch statin dosing for their patients in response to concerns about those side effects. The idea behind this instrument, then is that a patient with hypercholestrolemia who sees a doctor who tends, all else equal, to switch dosing more frequently for their other patients should be more likely to receive a recommendation of dose switching for reasons that have nothing to do with non-liver related health outcomes. We use this IV in models where the endogenous right-hand-side variable is related to the statin dose a patient is taking.

We purge our calculation of both IVs of all mechanically induced correlation between the instrument and the outcome by excluding each patient's own contribution to his or her physician's IV value. This construction guarantees that our instruments are consistent with the SUTVA assumption of Imbens and Angrist (1994) 12

We use a binary version of our instrumental variable. To generate this binary instrument, we calculate the average value of the instrument as described above, and then assign a value of one if the physician's value is above the overall average and zero otherwise. This approach may be understood as a comparison of each physician's value relative to the community norm. We find that empirically, our IVs correlate strongly

\footnotetext{
${ }^{12}$ The SUTVA implies that potential outcomes for each person $i$ are unrelated to the treatment status of other individuals (Angrist et al., 1996).
} 
with a patient's own behavior (the F-stat from first-stage suggested in Bound et al. (1995) is much greater than 10 for both our IVs).

One possible concern about the instruments are that the selection process that matches patients to physicians is not random, so perhaps patients with worse hypercholesterolemia choose physicians who, for instance, are known to provide higher dose statins. However, in the Italian primary care medical system, this sort of selection process is not plausible because the choice of GP is based on geographic proximity (see Section 3.1]. The local health authority assigns patients to GPs on the basis of proximity, and GPs cannot (and do not have any financial motive to) reject a patient assigned to them. Therefore, different values of our instrument are more likely to reflect practitioner ability and practice style, rather than unobserved differences in physician behavior due to the underlying health condition of the patient 13

\section{Results}

In this section, we present our empirical test of the necessary conditions (NCs) and the testable implications (TIs) summarized in Section 4.7 .

\subsection{Does Patient Adherence to Therapy Reduce Serum Cholesterol? - NC1}

In Table 4 we present the relationship between adherence to prescribed therapy (measured by the MPR) and the change in a patient's cholesterol level over a year. We estimate yearly log changes in LDL cholesterol $(Y)$ conditional on adherence above and below $75 \%(D=1(M P R>0.75)){ }^{14}$ Clinically, physicians use an LDL level above $2.58 \mathrm{mmol} / \mathrm{L}$ as a threshold to consider starting statin therapy, and reductions in LDL levels tend to reduce the risk of myocardial infarction, so even a change in $Y$ of 0.05 units can be clinically meaningful.

We estimated these effects for each statin molecule (rosuvastatin, simvastatin, and atorvastatin), as well as for each of the three treatment periods. Each cell in the table presents the key result (the coefficient on $D$ ) from each regression 15 We present both OLS and IV results, where the IV regressions employ the physician-level statin adherence variable that we describe in Section 5.2. Each cell in the OLS column reports $\beta_{D}=E[Y \mid D=1]-E[Y \mid D=0]$ from each regression, while each cell in the IV column should be

\footnotetext{
${ }^{13}$ Depalo (2018) provides further evidence in favor of this instrument and runs several falsification tests using a different vintage of the same dataset.

${ }^{14}$ Monaldi et al. (2015) suggests this threshold. Based on similar data, Atella et al. (2017) provides evidence on the robustness of our result to changes in this threshold.

${ }^{15}$ Full regression results are available upon request.
} 
interpreted as the local average treatment effect (LATE) of adherence on the change in LDL levels (please see Appendix A. Although potentially biased because of the endogeneity of adherence behavior, the OLS results are interesting because they represent an estimate of the population-level average treatment effect.

Overall, the results in Table 4 are consistent with our hypothesis NC1 that adherence to therapy leads to lower LDL cholesterol levels in the population at large. As expected given the randomized trial evidence, patients who more consistently adhere to statin therapy experience larger reductions in LDL cholesterol levels than patients who do not. All the results are statistically significant at the $p<0.05$ level, so we suppress the reporting of standard errors to reduce clutter in the table (these are available upon request). The OLS treatment effect is smaller during the last treatment period (3.5\% before 2006, about $2.5 \%$ between 2006 and 2011 and $1.5 \%$ after 2011). This result is interesting because it is consistent with the idea that the share of patients under statin treatment expanded among those in worse unobservable (to us) health condition as the fear of liver damage diminished.

As with the OLS results, the IV results show that adherent patients see a greater decrease in their LDL cholesterol than non-adherent patients. The LDL reduction from IV is never smaller than that obtained with OLS, thus supporting the idea that the latter is negatively biased due to self-selection. Under the LATE interpretation, this treatment effect estimate applies to the set of patients who adhere to therapy because they are seeing a doctor whose other patients adhere at a rate higher than the overall adherence rate in the population, but would not adhere otherwise. As a consequence, when we test the predictions of our model we should properly distinguish among various subpopulations (see Section 6.4. 16

\subsection{Do Higher Prescribed Doses Reduce Serum Cholesterol? - NC2}

In this section, we explore the effect of an increase in the statin dose on serum LDL cholesterol levels. In particular, we compare the change in serum LDL among patients who had an increase in their prescribed dose relative to the set of patients how had no change in their prescribed dose. Hence, we exclude patients who had a decrease in dose from the analyses we report in this subsection. Our results are presented in Table 5 . which is structured similarly to Table 4 except that the key right-hand side variable is a dummy variable indicating whether the equipotency dose of the statin was increased during the year. Each entry in Table 5 represents the treatment effect of a higher prescribed dose on serum LDL levels measured in mmol/L (with the OLS results showing a biased estimate of the population average treatment effect and the IV showing

\footnotetext{
${ }^{16}$ In order to obtain the population average treatment effect we also repeat the analysis using bounds robust to endogeneity, and valid for the entire population (Manski, 1990 Bhattacharya et al. 2008). For simplicity, we do not present these results, but they are available upon request and are consistent with those discussed here.
} 
a consistent estimate of the local average treatment effect). We do not report standard errors in this table because all the results are statistically significant at the $p<0.05$ level for all the entries in the table. ${ }^{17}$ In one sense, one might think that the answer to this question is available from the numerous randomized trial data, which show that increasing statin dose leads to substantial reductions in serum cholesterol levels reported in Table 1. However, the real-world evidence is complicated by two phenomena not tackled by the RCTs. First, the set of people who are treated in the population include many patients who have health conditions that were not included in the trials (Deaton and Cartwright, 2018). Though subsequent RCTs may be conducted that expand the set of patients exposed to treatment (such as the GREACE trial which included patients with liver disease), physicians are free to prescribe statins to patient groups not represented in the trial (and often do). Thus, the treatment effect in the population may differ from what the randomized trials report.

Second, the set of people who participate in the randomized trials are typically much more likely to adhere to prescribed therapy than are patients in the population at large (Frolkis et al., 2002). In trials, investigators regularly remind patients to stick to the therapy they have been randomly assigned. Low adherence presents problems for investigators in calculating the treatment effect of the drug, so they take actions to increase adherence. This is less common in real-world treatment settings, where patients make their own decisions about whether to follow their doctors' recommendations. Analogously, in real-world settings, patients may engage in a form of moral hazard. In particular, they may respond to an increase in statin dose by consuming a worse diet or decreasing their exercise regimen since they feel more protected against heart disease due to the statin.

Except for simvastatin (the oldest of the statin molecules), in Table 5 the OLS results before 2011 show an increase in mean LDL cholesterol levels after an increase in the dose of statins. These results are inconsistent with the conclusions from the RCT evidence, which show a decrease in LDL levels following increases in statin dose. By contrast, the OLS result shows the expected decline in LDL levels after 2011 for all three molecules. One possible interpretation of these pre 2011 results is that the OLS coefficients are biased. A second possible interpretation is that before 2011, patients responded on average to a recommendation of increasing dose by reducing their adherence to the medication due to fear of worse side effects. A third possible interpretation is that before 2011, Italian doctors only prescribed higher doses of statins to patients in worse initial unobserved health.

\footnotetext{
${ }^{17}$ We use a physician-level propensity to recommend dose switches to their other patients as an instrumental variable. In particular, we use a dummy indicator equal to one when the physician has a dose switching frequency higher than the average across all physicians, where the specific physician's average is computed net of the effect of the single patient.
} 
By contrast, the IV results, which are robust to unobserved health differences between patients on higher and lower statin doses, confirm for the RCT evidence. When physicians change the prescribed dose, then, compliers (in the LATE sense) enjoy decreases in serum LDL 18

Over time, the IV results in Table 5 are remarkably stable. Notice, though that the effectiveness of changing the dosage as estimated by IV decreases slightly over time. This fact may imply that the subpopulation of compliers includes patients with worse health characteristics in later periods. Since physicians prescribe the medication, having an in-depth look into the subpopulation of compliers is a first important test on how practitioners changed their behavior, based upon the new evidence. We present this analysis in the next section. The results in Table 5 do not necessarily imply that increasing the effective dose would always be effective, thus suggesting that all physicians should prescribe higher doses to their patients. Rather, these results imply that physicians are good at predicting when an increase in the prescribed dose would not lead a patient to adhere less to therapy, thus gaining the full benefit from switching to a higher dose.

Tables 4 and 5 together imply that the necessary conditions for our model are met. We turn next to the testable implications (TI).

\subsection{Do Physicians Learn Through Their Patients? - TI1}

To test whether doctors learn from their practice, we examine whether doctors who treat larger numbers of patients with high LDL levels show evidence of updating their beliefs about the side effects of statins, such that these beliefs become closer to the truth even before the GREACE and the revision of the scientific evidence. The key idea behind our test is that, if physicians do exhibit this type of learning, physicians with large practices should be less willing to attribute an abnormal liver function test in their patients to statins. Thanks to their learning they understand that the abnormal values are not the consequence of the statins (from Section 4 recall that $\left.g^{\prime}(N) \geq 0\right)$.

To check this prediction, we divide up the physicians in our samples based upon the quartiles of the number of patients treated with statins in 2014. Doctors in the first quartiles treat the fewest patients, while doctors in the fourth quartile treat the most. In Table 6, we report the mean number of liver function tests per patient per quarter, by treatment period and by quartile of practice size. We limit the sample in this

\footnotetext{
${ }^{18}$ Even if the reader is skeptical about our choice of instrument, we argue that a violation of the IV assumption would likely tend to produce an attenuation bias on our results in this specific analysis. Suppose that the physician's propensity to change the dose is correlated with a patient's unobserved health status. In the case of statins, one of the few reasons why a physician might recommend switching to an alternate therapy is that the current molecule and dose is not producing a sufficient reduction in LDL cholesterol level. Thus switches will be most likely to occur (and physician propensity to switch will be the highest) when the observed change in cholesterol level is the smallest. Even in this case, our results are confirmed for the population at large, using bounds as in Bhattacharya et al. (2008). These are available upon request.
} 
subsection to the set of patients who were prescribed either a higher or lower dose of statins over the year and exclude those who had no change in dose since practice guidelines suggested liver function testing with an increase in dose or switch of molecule. We analyze the set of patients who were prescribed a higher dose separately from those prescribed a lower dose. Moving from the first to the fourth quartile, we find that the number of prescribed tests decreases across all periods.

The first thing to notice in this table is that the number of liver function tests is substantially lower than one might expect from reading practice guidelines from the 2010 era and before. The highest value among the averages presented is only 0.282 tests among patients prescribed an increase in dose in 2006, which means most such patients received fewer than a single liver function test every quarter. Clearly, the guideline recommendations on liver function testing were not followed to the letter. Second, the rate of testing among patients whose dose was increased during the year was between 15 to 20 times than it was among patients whose dose was decreased. This fact is entirely consistent with the idea that physicians worried more about hepatic side effects from increasing statin dose than they did from decreasing it. This remains true even after 2010 after the release of the GREACE trial. Third, the testing rate declined over time in all quartiles of physician volume. This result again is consistent with physicians following the implications of the evolving statin literature, even before guidelines changed.

Finally, when we compare the number of tests per patient in smaller versus larger practices (i.e. move from the first to the fourth quartile), we find - consistent with TI1 of our theoretical model - that physicians who treated more patients tended to order fewer liver function tests for patients who were prescribed an increase in dose. This was true even before 2006, before GREACE and the observational studies that established the hepatic safety of statin medications. This result is consistent with physician learning-bydoing. To make a step further with respect to our model, it is interesting to compare the magnitude of this learning-by-doing effect with the magnitude of the effect of the GREACE study. We go horizontally across the table to explore the learning-by-doing effect: before 2006, the smallest practices ordered 0.282 tests per patient, while the largest practices ordered 0.261 per patient - a difference of 0.021 tests per patient. There are similar differences in each of the three periods. Now, we go vertically down the table to explore the effect of GREACE: in the lowest physician volume quintile, for instance, the testing rate declines from 0.282 tests per patient before 2006 to 0.225 tests per patient after 2010 - a difference in 0.057 tests. There are similar differences in each quartile of patient volume. Roughly speaking, the release of GREACE was about three times more important than learning-by-doing, as far as hepatic testing behavior is concerned. 


\subsection{Does Physician Learning Shift Patients into Treatment? - TI2}

In this section, we discuss how the clinical characteristics of the treated population has changed over the treatment periods. Our model's prediction is that over time we should observe more patients with worse clinical characteristics treated with statins.

We divide up the patients into four groups based on the Charlson index, ranging from very healthy patients ("Charlson very low") to patients with many comorbid conditions ("Charlson high"). Table 7 presents the Charlson index for our patient sample 19 In addition to conditioning on the treatment period, we split the sample into four groups based on a binary instrument variable $(Z)$ (in this case, physician-level propensity to swtich statin dosing more than their peers, as described above), and on whether the doctor recommended a change in the equipotency level of the prescribed medication for the patient $(D) 20$ Based on the terminology by Angrist et al. (1996), these groups are:

- The "always-takers" (patients who had a change in equipotency dose despite being seen by a doctor who recommends equipotency changes less frequently than average);

- The "never-takers" (patients who do not have a switch in recommended dose, despite being seen by a doctor who recommends equipotency changes more frequently than average);

- The "compliers", a group that consists of two distinct types of patients: 1) patients who are seen by doctor who recommends equipotency changes more frequently than average and who have been recommended to switch dose; and 2) patients who are seen by doctor who recommends equipotency changes less frequently than average and who have not been recommended to switch dose. The first of these two "complier" groups include "always-takers" mixed with the actual compliers, while the second includes "never-takers" mixed in (Imbens and Rubin, 1997).

We split the sample into these four groups since they may behave differently in response to the changing evidence base. In this context, taking into account the possible endogenity of switching behavior is important because the actual beahvior depends on the interaction between patients and GPs. By contrast, hepatic testing behavior predicted by TI1 is typically an independent choice made by the GP alone (i.e. exogenous).

\footnotetext{
${ }^{19}$ We present only the Charlson index for the sake of brevity, although we considered a larger set of characteristics. The complete set of results, available upon request, includes: anemia prevalence, asthma prevalence, bodyweight or obesity prevalence, exercise habits, chronic obstructive pulmonary disease prevalence, HDL and LDL cholesterol levels, history of cardiac bypass surgery or balloon angioplasty, history of cancer, diabetes prevalence, atrial fibrillation prevalence, hypertension prevalence, ischemic heart disease prevalence, heart attack history, and history of vascular disease.

${ }^{20}$ Indeed our model's prediction does not depend on $Z$. We thus verify that all results hold without conditioning on it.
} 
Each entry in a column of Table 7 reports the fraction of patients in the sub-population indicated by Charlson group.

Two dimensions are relevant for a meaningful comparison: we can fix the period and compare different sub-population, or we can fix the sub-population and compare different periods. With respect to the former, on many important clinical and demographic characteristics, the "never-taker", "always-taker", and "complier" groups are indistinguishable from one another on average. This lack of differences across these groups can be summarized nicely by the fact that the distribution of Charlson index scores (Table 7) does not differ across the four groups in a clinically significant way.

One important consequence of this finding is that trying to develop a model to predict whether a doctor is likely to recommend a switch in therapy conditional on these observed clinical and demographic characteristics (perhaps using machine learning methods) would be a difficult challenge. There is clearly too much clinical overlap between the groups to allow an easily observable distinction. Of course, in a different setup, it may be perfectly possible to reach this goal (Currie and MacLeod, 2018). In our case, doctors recommend switches in equipotency dose based on characteristics that we may not observe, even though we observe so many clinical characteristics.

By contrast, looking at the results across patient types, Table 7 shows a substantial change in the clinical characteristics of the population in the direction of sicker patients entering statin treatment over time. For all subgroups of patients, health conditions become worse. For patients in worst health conditions ("Charlson index high") this is particularly true in the post-GREACE trial period.

The effect was stronger for patients that switched their therapy $(D=1)$, rather than for those who did not $(D=0)$. To see this consider the difference (for a given sub-population, over different periods) in difference (the former difference across different sub-population) parameter. For example, the share of patients in most critical conditions (high Charlson index), during the period 2006-2010 increased by $3(\approx 0.11-0.08)$ and $4(\approx 0.11-0.07)$ percentage points for never takers and non taker-compliers, respectively, with respect to the previous periods. This increase was 4 and 5 percentage points for takers-compliers and always takers, respectively. Therefore, the causal effect was equal to 1 percentage points ${ }^{21}$ By the same token (and consistent with the above finding) the share of patients in better condition (a very low Charlson index) decreased over time, and the reduction was larger for patients that switched their therapy rather than for those who did not.

The difference-in-difference calculation aside, the results in Table 7 are entirely consistent with TI2. As

\footnotetext{
${ }^{21}$ The conclusions hold even if we consider $D=(0,1)$, without taking into account the instrument $Z$.
} 
new information about the side effect profiles of statin became available, physicians updated their beliefs so that the set of patients under treatment expanded to include patients who were previously thought to be poor candidates for the statins. This was true for all the groups we examine (compliers, always-takers, and never-takers alike). Hence, TI2 is not falsified.

\subsection{Does Physician Learning Change Testing Behavior? - TI3}

We turn next to an examination of whether physicians are more likely to recommend a change in statin equipotency dose as a function of the number of liver tests a physician ordered (or has ordered) for a patient with respect to the previous quarter. These results are reported in Table 8 . For all patients (see the 'Overall' column), the probability of a change in therapy is an increasing function of the number of tests: for instance, patients who take at least four tests are more likely to switch than patients who have their liver function tested no more than three times in every time period. This is consistent with both the pre-GREACE guidelines and the post-GREACE American guidelines, which suggests testing liver functionality at the time of a recommended switch in therapy. As we saw in Table 6, relative to patients recommended a higher statin dose, patients who are recommended a lower dose undergo a larger number of liver function tests. On balance, these results suggest that Italian doctors followed these guidelines (see footnote 2).

Motivated by the information shock provided by GREACE, we examine changes across treatment periods, holding fixed switcher groups ("vertical" comparison). For all groups of patients, there is an abrupt change in "testing behavior" as new information arrives. For example, with respect to the baseline category of no liver function tests, the probability of switching the therapy to a lower class of equivalence with four tests was larger than 2\% (0.021) before 2006, compared with less than 2\% between 2006 and 2010 (0.018; a difference equal to $\sim-0.003)$ that further decreased to about $1 \%$ after GREACE (0.013). Similar behavior is observed for switches to higher equivalence class: the probability before 2006 was equal to $13.5 \%$ and $13.1 \%$ between 2006 and 2010 (a difference equal to $\sim-0.005$ ).

These results are consistent with the idea that Italian physicians changed their practice in response to new scientific information, in a relatively quick way. For all the groups we analyze, we cannot reject implication TI3 of our model that after the introduction of new clinical evidence, doctors changed their beliefs in the direction of the new findings in the literature.

At this point, we have all the components to enrich our analysis by computing a causal difference-indifference parameter (see Appendix B). From the previous example the causal effect is equal to 0.2 percentage points $(-0.002=-0.005-(-0.003))$. This estimate suggests that after the information shock, the number of tests 
decreased for both groups of patients (as suggested by the model). However, the decrease was larger for those switching to a higher dosage (consistent with the fact that the concern related to liver function damages decreased). Also, the decrease was larger after GREACE. By comparing Table 6 and Table 8 , we find that the largest change in physicians behavior took place after GREACE became publicly known rather than as a result of learning by doing since the DID effects implied by the latter table are larger than those implied by the former.

\subsection{Did Physicians Prescribe Higher Doses in Response to the Information Shock? - TI4}

In Figure 1, we investigate the statin prescription behavior of the physicians with respect to equipotency dose. Between 2005 and 2006, there was a steep increase in the dosage and strength of statins prescriptions, followed by a further increase between 2010 and 2011 .

In Figure 2, we show this same figure, except we distinguish between different groups of patients with higher and lower Charlson scores (that is, between patients with greater or fewer comorbid conditions). This figure shows a pattern similar to those seen in Figure 1 for all patients groups. The increase in dosing is similar over this period for all four groups (roughly 0.25 units, which is equivalent, roughly to a $10 \mathrm{mg}$ increase in the dose of Simvastatin). The results in these figures are entirely consistent with TI4 of our model: physicians indeed prescribe higher equipotency in response to new information on statin safety, even for their sickest patients with many other comorbid conditions.

\subsection{Does Physician Learning Increases Adherence? - TI5}

In Table 9, we examine how patient adherence to therapy $(M P R>0.75)$ as well as HDL, LDL, and total cholesterol outcomes (all measured in $\mathrm{mmol} / \mathrm{L}$ ) changed over time for different subpopulations of patients. Like in Table 8, we divide up the sample into four groups ("Never", " $D=0$ Compliers", " $D=1$ Compliers", and "Always") on the basis of a binary instrumental variable $(Z)$, and on whether the doctor recommended a change in the equipotency level of the prescribed medication for the patient $(D)$. As in Table 8 we use physician-level propensity to recommend statin dose changes to their other patients as our instrumental variable (please see Section 5.2 . As before, we interpret the groups as follows:

- "Never" represents the set of patients who would not have been recommended a change in statin dose regardless of whether they are seeing a doctor who tends to recommend dose changes more often than 
average for their other patients;

- "Always" represents the set of patients who would have been recommended a dose change even if seeing a doctor recommends dose changes at lower than average rate;

- " $D=0$ Compliers" represents the set of patients who were not recommended a dose change by doctors who tend to recommend dose changes at a lower than average rate among their other patients; and

- " $D=1$ Compliers represents the set of patients who were recommended a dose change and saw a doctor who recommends dose changes at a higher than average rate.

The most striking finding is that adherence to therapy increased over time for all four types of patients. This result confirms our reading that the adherence rate increased following the spread of information provided by GREACE and before, perhaps because physicians became more confident in telling patients that the risk of side effects was low. The size of this effect is substantial - for all four groups, adherence increased by at least 12 percentage points between the "Pre" 2006 and "Post" 2010 periods. In fact, nearly all the increase in adherence occurred before the release of the GREACE trial, during a period where it was primarily non-randomized studies were published addressing the possibility of liver side effects from statins.

The bottom three rows of Table 9 show changes in HDL, LDL, and total cholesterol in the four populations over time. While HDL cholesterol declined only modestly, LDL cholesterol levels and total cholesterol levels declined substantially over this period. LDL and total cholesterol levels decline consistently throughout this whole period.

\section{Conclusion}

In this paper, we investigate the relationship between technological advance, in the form of an information shock, and physician and patient behavior. We focus on the case of statins, a medication used to manage high cholesterol levels, where newly produced evidence has altered the scientific consensus regarding side effect profiles. Early common wisdom held that statins might cause liver damage, and therefore, physicians should regularly test their patients for changes in liver enzyme levels. In 2010 GREACE, a large and influential randomized controlled trial, suggested instead that the medication could be continued despite elevated liver enzymes, and that patients need not be tested regularly for liver damage. We exploit this major informational, technological shock to test how physician prescription and testing behavior, as well as patients adherence to therapy, changed in response. 
We test our model using a unique dataset representative of the Italian population, that links patients to doctors over the period 2003-2014. We account for the possible non-random sorting of patients into treatment in our estimates. Our main conclusion is that doctors responded promptly to this technological shock by decreasing unnecessary liver function testing of patients. We also find that even before the shock occurred, physicians with larger practices had already adopted clinical practices consistent with the information generated by the study. Along the way, we also show that statins, as prescribed by physicians in the Italian setting for the Italian population, are effective in reducing serum cholesterol levels.

Broadly taken, our results suggest that investments in scientific knowledge can rapidly diffuse into practice in the context of a profession where continuing education is the norm. In this case, the technological shock was an information shock that changed the way a widely available technology (statin medication) is used, but differentially so in smaller practices. Larger practices had already learned at least part of the lessons of the GREACE trial before the trial was conducted and the trial results released.

As a general matter, understanding when information shocks of the sort we have analyzed will change practice will depend upon the nature, prominence, and importance of the technological innovation in question, as well as the incentives faced by professionals to learn about these innovations. Understanding when an information shock is likely to be effective is important for science policy, since it guides which expensive research projects are worthwhile for funding agencies to support. Proposed research projects that are more likely to change practice are presumably worthier of support than projects that would have no effect on real-world practice whatever the outcome. Thus, we encourage further developments in this literature that seek to test the conditions under which our general conclusions do or do not hold. 


\section{Appendix A Population Average Treatment Effect}

We employ the standard framework of potential outcomes, in which the outcome $Y$ (such as reduction of cholesterol) is observed either under treatment $\left(Y_{1}\right)$ or under non-treatment $\left(Y_{0}\right)$, but not both. In this notation, the subscript on $Y$ indicates treatment status $(d \in D$ takes value 1 for treatment and 0 for non-treatment). The parameter of interest is the population average treatment effect (ATE):

$$
\begin{aligned}
\Delta & =E\left[Y_{1}\right]-E\left[Y_{0}\right] \\
& =E\left[Y_{1} \mid D=1\right] P[D=1]+E\left[Y_{1} \mid D=0\right] P[D=0] \\
& -E\left[Y_{0} \mid D=1\right] P[D=1]+E\left[Y_{0} \mid D=0\right] P[D=0] .
\end{aligned}
$$

If we could observe the outcome under the two states of the world for a randomly chosen individual, the identification of ATE would be straightforward. In fact, the sampling process is informative only about the outcome under treatment for treated individuals $\left(E\left[Y_{1} \mid D=1\right]\right)$ and about the outcome under non-treatment for non-treated individuals $\left(E\left[Y_{0} \mid D=0\right]\right)$. As standard in this literature, the main challenge in the analysis is to recover the outcome for treated patients had they not been treated, or $E\left[Y_{1} \mid D=0\right]$, and vice versa for non-treated patients.

In order to allow for possible non-random sorting into treatment, we represent the average treatment effect in terms of the following index function model (Vytlacil, 2002):

$$
\begin{aligned}
Y_{d} & =r(D)+\epsilon_{d} \\
D^{*} & =s(Z)-v \quad D=1\left(D^{*}>0\right),
\end{aligned}
$$

where $D^{*}$ is the selection rule that determines who is treated on the basis of a binary variable $Z$ (our instrument) which determines treatment, but not the outcome and where $\epsilon_{d}$ and $v$ are unobservable disturbances.

We divide the discussion of our empirical methods based on which assumptions we are willing to impose on the relation between $\epsilon_{d}$ and $v$. In the first part, we discuss the methods to recover the population average treatment effect under assumptions of exogeneity (ordinary least squares). In the second part, we discuss methods to recover the local average treatment effect (LATE) if treatment assignment is endogenous (instrumental variables methods). 


\section{Recovering the ATE under exogeneity}

In the simplest case, when $\epsilon_{d} \perp v$ we have that $E\left[Y_{d} \mid D=0\right]=E\left[Y_{d} \mid D=1\right]$. It then follows that $E\left[Y_{1} \mid D=\right.$ $0]=E\left[Y_{1} \mid D=1\right]$ and $E\left[Y_{0} \mid D=0\right]=E\left[Y_{0} \mid D=1\right]$, which can be substituted in equation 18 to derive $\Delta=E\left[Y_{1} \mid D=1\right]-E\left[Y_{0} \mid D=0\right]$. This parameter is valid for the entire population, but it is biased if treatment assignment is endogenous (that is, doctors assign treatment based on information they observe but that we do not). As such, it is incoherent with our economic model. We present these results as baseline model to compare our subsequent estimations that measure the effect of the treatment while taking into account the endogenous treatment assignment.

\section{Recovering the LATE under endogeneity}

A major theme of Section 4 is that the sample of treated individuals is not completely at random (in the terminology of Little, 1995). Physicians recommend therapy on the basis of variables that both researchers and patients observe ("observables"), as well as on variables that they observe, but researchers do not ("unobservables").

Suppose we have an instrument, $Z$, in the sense described in equation 19, such that (without loss of generality) $Z$ and $D$ are positively correlated, and the following conditions are met Imbens and Angrist 1994, Angrist et al. 1996):

1. potential outcomes are unrelated to the treatment status of other workers (also known as stable unit treatment value assumption, or SUTVA);

2. the instrument is correlated with the treatment indicator, $D$;

3. the instrument does not affect the potential outcome (that is, $Z$ does not affect $Y_{d}$ in equation 19), and

4. monotonicity, so that $D_{1} \geq D_{0}$ for each patient, where $D_{z}$ is the potential treatment with the instrument $Z=z$. Under this assumption, there are exactly three groups: "always takers" who are always treated no matter the value of $Z$, "never takers" who are never treated no matter the value of $Z$, and "compliers" who are treated if $Z=1$ and are not treated if $Z=02$

\footnotetext{
${ }^{22}$ A fourth group, known as "defiers" is made by those who do the opposite with respect to the assignment of the instrument, i.e. $\{Z=d, D=1-d\}$. This group would violate the monotonicity condition, whence its share in the population is identically 0 .
} 
Under these hypotheses, we identify

$$
L A T E \equiv \frac{E\left[Y_{1} \mid Z=1\right]-E\left[Y_{0} \mid Z=0\right]}{E\left[D_{1} \mid Z=1\right]-E\left[D_{0} \mid Z=0\right]}=E\left[Y_{1}-Y_{0} \mid D_{1}>D_{0}\right]
$$

Under heterogeneous effect of the treatment, the IV estimator identifies a local average treatment effect ( $L A T E)$, valid for the subpopulation of patients who are induced to be treated by a change in the instrument, or compliers (Angrist et al., 1996). Although patients belonging to this "complier" subpopulation cannot be identified on the basis of the observable covariates, the distribution of their characteristics can be described Angrist, 2004) ${ }^{23}$ As a consequence, even though our model provides a fairly large number of theoretical predictions, the analysis of the compliers will be an important exercise for coherency between the data and the model beyond the test of the necessary conditions themselves.

\section{Appendix B Causal interpretation of the estimates}

Once verified the NCs required by our theoretical model in Section 4 the next step is to obtain a causal interpretation of all estimates stemming from the TIs. This becomes a relatively simple task once we realize that all our TIs are based on comparison of estimates obtained from two subpopulations (individuals who switch the therapy as opposed to those who do not) observed for two periods, before and after the shock occurs. This represents a standard Difference-in-Difference (DID) setup, which delivers estimates that can be interpreted as causal. For a better understanding of this causal framework, let us define the outcome $Y$ as $Y_{D T}$, where $\mathrm{D}=1$ for patients who switch their therapy and $\mathrm{D}=0$ otherwise, and $\mathrm{T}=0$ for the reference period and $\mathrm{T}=1$ for the 'treatment' period (i.e., $\mathrm{T}=0$ before 2006 and $\mathrm{T}=1$ between 2006 and 2010; $\mathrm{T}=0$ between 2006 and 2010 and $T=1$ after 2010). We first compute the difference between $T=1$ and $T=0$ for patient who switch their therapy (or treated, for brevity), i.e. $\Delta Y_{1}=Y_{11}-Y_{10}$; we do the same for patients who do not switch their therapy (untreated), i.e. $\Delta Y_{0}=Y_{01}-Y_{00}$. The difference $\left(\Delta Y_{1}-\Delta Y_{0}\right)$ is then equivalent to a DID estimator, that is the causal effect of the information shock on the outcome $Y{ }^{24}$

\footnotetext{
${ }^{23}$ IV-LATE would identify the population ATE if the treatment effect of statins can be assumed homogeneous within the whole population, or when the entire population consists of compliers, or patients do not self-select on the basis of their idiosyncratic returns (Heckman, 1997. Blundell et al. 2005), or some external information excludes specific subpopulations (Oreopoulos, 2006 ).

${ }^{24} \mathrm{We}$ have verified that the common trend assumption is satisfied (the results are available upon request).
} 


\section{References}

Angrist, J. D. (2004). Treatment effect heterogeneity in theory and practice*. The Economic Journal 114(494), C52-C83.

Angrist, J. D., G. W. Imbens, and D. B. Rubin (1996). Identification of causal effects using instrumental variables (Disc: p456-472). Journal of the American Statistical Association 91, 444-455.

Atella, V., F. Belotti, C. Bojke, A. Castelli, K. Grai, J. Kopinska, A. P. Mortari, and A. Street (2019). How health policy shapes healthcare sector productivity? evidence from italy and uk. Health Policy 123(1), $27-36$.

Atella, V., F. Belotti, and D. Depalo (2017, sep). Drug therapy adherence and health outcomes in the presence of physician and patient unobserved heterogeneity. Health Economics 26, 106-126.

Atella, V. and V. Conti (2014). The effect of age and time to death on primary care costs: The italian experience. Social Science \& Medicine 114, 10-17.

Atella, V. and F. D'Amico (2015). Who is responsible for your health: is it you, your doctor or the new technologies? The European Journal of Health Economics 16, 835-846.

Atella, V. and J. A. Kopinska (2014, Apr). The impact of cost-sharing schemes on drug compliance in italy: evidence based on quantile regression. International Journal of Public Health 59(2), 329-339.

Athyros, V. G., K. Tziomalos, T. D. Gossios, T. Griva, P. Anagnostis, K. Kargiotis, E. D. Pagourelias, E. Theocharidou, A. Karagiannis, and D. P. Mikhailidis (2010, dec). Safety and efficacy of long-term statin treatment for cardiovascular events in patients with coronary heart disease and abnormal liver tests in the Greek Atorvastatin and Coronary Heart Disease Evaluation (GREACE) Study: a post-hoc analysis. The Lancet 376(9756), 1916-1922.

Avdic, D., S. von Hinke Kessler Scholder, B. Lagerqvist, C. Propper, and J. Vikstrom (2018). Information shocks and provider adaptation: Evidence from interventional cardiology. Technical report.

Bays, H. (2006). Statin Safety: An Overview and Assessment of the Data-2005. American Journal of Cardiology $97(8$ SUPPL. 1).

Bays, H., D. E. Cohen, N. Chalasani, and S. A. Harrison (2014). An assessment by the Statin Liver Safety Task Force: 2014 update. Journal of Clinical Lipidology 8(3 SUPPL), S47-57. 
Bhardwaj, S. S. and N. Chalasani (2007, aug). Lipid-Lowering Agents That Cause Drug-Induced Hepatotoxicity. Clinics in Liver Disease 11(3), 597-613.

Bhattacharya, J., A. M. Shaikh, and E. Vytlacil (2008, May). Treatment Effect Bounds under Monotonicity Assumptions: An Application to Swan-Ganz Catheterization. American Economic Review 98(2), 351-56.

Blundell, R., L. Dearden, and B. Sianesi (2005). Evaluating the effect of education on earnings: models, methods and results from the national child development survey. Journal of the Royal Statistical Society: Series A (Statistics in Society) 168(3), 473-512.

Bound, J., D. Jaeger, and R. Baker (1995). Problems with instrumental variable estimation when the correlation between the instruments and the endogenous explanatory variable is weak. Journal of the American Statistical Association 90, 443-450.

Bradford, R. H., C. L. Shear, A. N. Chremos, C. Dujovne, M. Downton, F. A. Franklin, A. L. Gould, M. Hesney, J. Higgins, and D. P. Hurley (1991, jan). Expanded Clinical Evaluation of Lovastatin (EXCEL) study results. I. Efficacy in modifying plasma lipoproteins and adverse event profile in 8245 patients with moderate hypercholesterolemia. Archives of internal medicine 151(1), 43-9.

Calderon, R. M., L. X. Cubeddu, R. B. Goldberg, and E. R. Schiff (2010, apr). Statins in the treatment of dyslipidemia in the presence of elevated liver aminotransferase levels: a therapeutic dilemma. Mayo Clinic proceedings $85(4), 349-56$.

Charlson, M., P. Pompei, K. Ales, and C. R. MacKenzie (1987). A new method of classifying prognostic comorbidity in longitudinal studies: development and validation. J Chron Dis 40(5), 373-383.

Clarke, A. and P. Mills (2006, oct). Atorvastatin associated liver disease. Digestive and Liver Disease 38(10), $772-777$.

Cohen, D. E., F. A. Anania, N. Chalasani, and National Lipid Association Statin Safety Task Force Liver Expert Panel (2006, apr). An Assessment of Statin Safety by Hepatologists. The American Journal of Cardiology 97(8), S77-S81.

Conforti, A., L. Magro, U. Moretti, S. Scotto, D. Motola, F. Salvo, B. Ros, and R. Leone (2006). Fluvastatin and hepatic reactions: a signal from spontaneous reporting in Italy. Drug safety 29(12), 1163-72.

Cramer, J., A. Roy, A. Burrell, and ... (2008). Medication compliance and persistence: Terminology and definitions. Value in Health 11. 
Currie, J. and W. B. MacLeod (2018). Understanding Physician Decision Making: The Case of Depression. National Bureau of Economic Research Working Paper Series No. 24955.

Deaton, A. and N. Cartwright (2018, aug). Understanding and misunderstanding randomized controlled trials. Social science E medicine (1982) 210, 2-21.

Depalo, D. (2018, April). Explaining the relation between adherence to medication and cholesterol through the marginal patient. Working paper.

Fabiani, L., M. Scatigna, K. Panopoulou, A. Sabatini, E. Sessa, F. Donato, M. Marchi, R. Nardi, C. Niccolai, F. Samani, and G. Ventriglia (2004). Health search: istituto di ricerca della societá italiana di medicina generale: la realizzazione di un database per la ricerca in medicina generale. Epidemiol and Prev 28, $156-162$.

Fichera, E., J. Banks, L. Siciliani, and M. Sutton (2018, nov). Does patient health behaviour respond to doctor effort? Journal of Economic Behavior \& Organization (62).

Frolkis, J. P., G. L. Pearce, V. Nambi, S. Minor, and D. L. Sprecher (2002, dec). Statins do not meet expectations for lowering low-density lipoprotein cholesterol levels when used in clinical practice. The American Journal of Medicine 113(8), 625-629.

Gafni, A., C. Charles, and T. Whelan (1998, aug). The physician-patient encounter: the physician as a perfect agent for the patient versus the informed treatment decision-making model. Social science $\mathcal{E}$ medicine (1982) 47(3), 347-54.

Gillett, R. C. and A. Norrell (2011, mar). Considerations for safe use of statins: liver enzyme abnormalities and muscle toxicity. American family physician 83(6), 711-6.

Heckman, J. (1997). Instrumental variables: A study of implicit behavioral assumptions used in making program evaluations. The Journal of Human Resources 32(3), 441-462.

Hughes, D., A. Bagust, A. Haycox, and T. Walley (2001). The impact of non-compliance on the costeffectiveness of pharmaceuticals: a review of the literature. Health Economics 10, 601-615.

Imbens, G. W. and J. D. Angrist (1994, March). Identification and Estimation of Local Average Treatment Effects. Econometrica 62(2), 467-75. 
Imbens, G. W. and D. B. Rubin (1997). Estimating Outcome Distributions for Compliers in Instrumental Variables Models. The Review of Economic Studies 64(4), 555-574.

Khorashadi, S., N. K. Hasson, and R. C. Cheung (2006, jul). Incidence of Statin Hepatotoxicity in Patients With Hepatitis C. Clinical Gastroenterology and Hepatology 4(7), 902-907.

Lewis, J. H., M. E. Mortensen, S. Zweig, M. J. Fusco, J. R. Medoff, R. Belder, and Pravastatin in Chronic Liver Disease Study Investigators (2007, nov). Efficacy and safety of high-dose pravastatin in hypercholesterolemic patients with well-compensated chronic liver disease: Results of a prospective, randomized, double-blind, placebo-controlled, multicenter trial. Hepatology 46(5), 1453-1463.

Little, R. J. A. (1995). Modeling the drop-out mechanism in repeated-measures studies. Journal of the American Statistical Association 90(431), 1112-1121.

Manski, C. F. (1990, May). Nonparametric Bounds on Treatment Effects. American Economic Review 80(2), $319-23$.

Maron, D., S. Fazio, and M. Linton (2000). Current perspectives on statins. Circulation 101, 207-213.

Mazzaglia, G., E. Ambrosioni, M. Alacqua, A. Filippi, E. Sessa, V. Immordino, C. Borghi, O. Brignoli, A. P. Caputi, C. Cricelli, and L. G. Mantovani (2009). Adherence to antihypertensive medications and cardiovascular morbidity among newly diagnosed hypertensive patients. Circulation 120(16), 1598-1605.

McKenney, J. M., M. H. Davidson, T. A. Jacobson, J. R. Guyton, and National Lipid Association Statin Safety Assessment Task Force (2006, apr). Final Conclusions and Recommendations of the National Lipid Association Statin Safety Assessment Task Force. The American Journal of Cardiology 97(8), S89-S94.

Mölgaard, J., B. L. Lundh, H. von Schenck, and A. G. Olsson (1991, dec). Long-term efficacy and safety of simvastatin alone and in combination therapy in treatment of hypercholesterolaemia. Atherosclerosis 91 Suppl, S21-8.

Monaldi, B., G. Bologna, G. G. Costa, C. D’Agostino, F. Ferrante, M. Filice, A. M. Grion, A. Mingarelli, L. Paloscia, R. Tettamanti, C. Veronesi, and L. Degli Esposti (2015). Adherence to statin treatment following a myocardial infarction: an Italian population-based survey. ClinicoEconomics and outcomes research : CEOR 7, 273-80.

Oreopoulos, P. (2006, March). Estimating average and local average treatment effects of education when compulsory schooling laws really matter. American Economic Review 96(1), 152-175. 
Pastori, D., L. Polimeni, F. Baratta, A. Pani, M. Del Ben, and F. Angelico (2015, jan). The efficacy and safety of statins for the treatment of non-alcoholic fatty liver disease. Digestive and Liver Disease $47(1)$, $4-11$.

Plakogiannis, R. and H. Cohen (2007, jan). Optimal low-density lipoprotein cholesterol lowering-morning versus evening statin administration. The Annals of pharmacotherapy 41(1), 106-10.

Rzouq, F. S., M. L. Volk, H. H. Hatoum, S. K. Talluri, R. R. Mummadi, and G. K. Sood (2010, aug). Hepatotoxicity Fears Contribute to Underutilization of Statin Medications by Primary Care Physicians. The American Journal of the Medical Sciences 340(2), 89-93.

Scandinavian Simvastatin Survival Study Group (1994, nov). Randomised trial of cholesterol lowering in 4444 patients with coronary heart disease: the Scandinavian Simvastatin Survival Study (4S). The Lancet $344(8934), 1383-1389$.

Scott, A. and S. Vick (1999, may). Patients, Doctors and Contracts: An Application of Principal-Agent Theory to the Doctor-Patient Relationship. Scottish Journal of Political Economy 46(2), 111-134.

Segarra-Newnham, M., D. Parra, and E. M. Martin-Cooper (2007, jun). Effectiveness and Hepatotoxicity of Statins in Men Seropositive for Hepatitis C Virus. Pharmacotherapy 27(6), 845-851.

Sniderman, A. D. (2004, nov). Is there value in liver function test and creatine phosphokinase monitoring with statin use? The American Journal of Cardiology 94(9), 30-34.

Vytlacil, E. (2002, jan). Independence, Monotonicity, and Latent Index Models: An Equivalence Result. Econometrica $70(1), 331-341$. 
Table 1: Statin Equipotency Dosing Conversion

\begin{tabular}{c|c|c|c|c}
\hline Equipotency Class & \% LDL reduction & Simva. & Ator. & Rosu. \\
\hline 1 & $<24$ & 5 & - & - \\
2 & $25-32$ & 10 & - & - \\
3 & $31-39$ & 20 & 10 & - \\
4 & $37-45$ & 40 & 20 & 5 \\
5 & $48-52$ & 80 & 40 & 10 \\
6 & $55-60$ & - & 80 & 20 \\
7 & $60-63$ & - & - & 40 \\
\hline
\end{tabular}

Table 2: Descriptive Statistics

\begin{tabular}{l|rrr|rrr}
\hline & \multicolumn{3}{|c}{ Women } & \multicolumn{3}{c}{ Men } \\
\hline Variable & Mean & S.D. & Median & Mean & S.D. & Median \\
\hline$\Delta$ Cholesterol & -0.038 & 0.192 & -0.019 & -0.038 & 0.185 & -0.020 \\
LDL & 120.701 & 36.269 & 115.779 & 110.847 & 34.909 & 106.314 \\
Adherence & 0.643 & 0.286 & 0.629 & 0.696 & 0.281 & 0.674 \\
ALP & 0.061 & 0.256 & 0.000 & 0.052 & 0.243 & 0.000 \\
ALT & 0.290 & 0.509 & 0.000 & 0.290 & 0.516 & 0.000 \\
AST & 0.272 & 0.496 & 0.000 & 0.270 & 0.502 & 0.000 \\
Albumin & 0.029 & 0.177 & 0.000 & 0.030 & 0.181 & 0.000 \\
Birilubin & 0.022 & 0.154 & 0.000 & 0.022 & 0.156 & 0.000 \\
Birilubin (Tot. \& Fract.) & 0.042 & 0.216 & 0.000 & 0.043 & 0.223 & 0.000 \\
Charlson index & 1.009 & 1.159 & 1.000 & 1.220 & 1.269 & 1.000 \\
Equipotency & 4.492 & 0.650 & 4.000 & 4.615 & 0.694 & 5.000 \\
Diabetes & 0.247 & 0.431 & 0.000 & 0.297 & 0.457 & 0.000 \\
Hypertension & 0.721 & 0.449 & 1.000 & 0.683 & 0.465 & 1.000 \\
Congestive heart failure & 0.027 & 0.162 & 0.000 & 0.039 & 0.192 & 0.000 \\
Atrial fibrillation & 0.051 & 0.219 & 0.000 & 0.063 & 0.242 & 0.000 \\
Vascular Diseases & 0.010 & 0.100 & 0.000 & 0.019 & 0.138 & 0.000 \\
PCI & 0.001 & 0.036 & 0.000 & 0.005 & 0.071 & 0.000 \\
Ischemic heart & 0.014 & 0.117 & 0.000 & 0.017 & 0.130 & 0.000 \\
Other Heart & 0.136 & 0.343 & 0.000 & 0.310 & 0.463 & 0.000 \\
Age & 68.947 & 9.312 & 70.000 & 65.539 & 10.281 & 66.000 \\
North West & 0.244 & 0.429 & 0.000 & 0.269 & 0.443 & 0.000 \\
Nort East & 0.211 & 0.408 & 0.000 & 0.215 & 0.411 & 0.000 \\
Center & 0.183 & 0.386 & 0.000 & 0.173 & 0.378 & 0.000 \\
South & 0.245 & 0.430 & 0.000 & 0.239 & 0.426 & 0.000 \\
Simvastatin & 0.441 & 0.496 & 0.000 & 0.400 & 0.490 & 0.000 \\
Atorvastatin & 0.342 & 0.474 & 0.000 & 0.383 & 0.486 & 0.000 \\
Rosuvastatin & 0.218 & 0.413 & 0.000 & 0.217 & 0.412 & 0.000 \\
\hline
\end{tabular}


Table 3: Number of Prescriptions by Active Ingredient and Dosage

\begin{tabular}{l|rrrr}
\hline & \multicolumn{3}{|c}{ Statin } \\
\hline Variable & mg & Simvastatin & Atorstatin & Rosustatin \\
\hline Men & 5 & 0 & 0 & 18705 \\
& 10 & 14282 & 105253 & 132127 \\
& 20 & 246066 & 166173 & 26579 \\
& 40 & 70926 & 42047 & 2455 \\
& 80 & 0 & 4131 & 0 \\
Women & 5 & 0 & 0 & 25440 \\
& 10 & 24684 & 137788 & 158079 \\
& 20 & 331071 & 164907 & 24892 \\
& 40 & 68835 & 24946 & 1684 \\
& 80 & 0 & 1525 & 0 \\
\hline
\end{tabular}

Table 4: Patient Adherence to Therapy Reduces Serum Cholesterol (NC1)

\begin{tabular}{l|cc}
\hline Statin & OLS & IV \\
\hline \hline \multicolumn{3}{c}{ Before 2006 } \\
\hline Simvastatin & -0.018 & -0.029 \\
Atorstatin & -0.026 & -0.021 \\
Rosustatin & -0.050 & -0.144 \\
\hline All & -0.034 & -0.058 \\
\hline \multicolumn{3}{c}{$2006-2011$} \\
\hline Simvastatin & -0.012 & -0.006 \\
Atorstatin & -0.020 & -0.023 \\
Rosustatin & -0.029 & -0.008 \\
\hline All After 2011 \\
\hline \multicolumn{3}{c}{ Aft } \\
\hline Simvastatin & -0.012 & -0.013 \\
Atorstatin & -0.023 & -0.030 \\
Rosustatin & -0.021 & 0.003 \\
\hline All & -0.017 & -0.028 \\
\hline
\end{tabular}

Note: The IV results use the indicator of above average physician-level high compliance rate $(M P R>0.75)$ among other patients as instrument. 
Table 5: Higher Prescribed Doses Reduce Serum Cholesterol (NC2)

\begin{tabular}{|c|c|c|}
\hline Statin & OLS & IV \\
\hline \multicolumn{3}{|c|}{ Before 2006} \\
\hline Simvastatin & -0.005 & -0.052 \\
\hline Atorstatin & 0.000 & -0.026 \\
\hline Rosustatin & 0.054 & 0.563 \\
\hline All & -0.005 & -0.065 \\
\hline \multicolumn{3}{|c|}{$2006-2011$} \\
\hline Simvastatin & -0.008 & -0.104 \\
\hline Atorstatin & 0.000 & -0.066 \\
\hline Rosustatin & 0.004 & -0.157 \\
\hline All & -0.005 & -0.055 \\
\hline \multicolumn{3}{|c|}{ After 2011} \\
\hline Simvastatin & -0.006 & -0.069 \\
\hline Atorstatin & -0.001 & -0.048 \\
\hline Rosustatin & -0.002 & -0.258 \\
\hline All & -0.008 & -0.035 \\
\hline $\begin{array}{l}\text { Note: The } \\
\text { ohysician-leve } \\
\text { erage dosing } \\
\text { nstrument. }\end{array}$ & & use \\
\hline
\end{tabular}

Table 6: Mean Number of Liver Function Tests by Quartile of Physician Patient Volume (TI1)

\begin{tabular}{|c|c|c|c|c|c|}
\hline & & \multicolumn{4}{|c|}{ Quartile of Patient Volume } \\
\hline & & $\begin{array}{l}1 \\
\text { (Low) }\end{array}$ & 2 & 3 & $\begin{array}{l}4 \\
\text { (High) }\end{array}$ \\
\hline \multirow{3}{*}{ Dose Reduction } & Before 2006 & 0.015 & 0.015 & 0.015 & 0.016 \\
\hline & 2006-2010 & 0.012 & 0.012 & 0.012 & 0.013 \\
\hline & After 2010 & 0.010 & 0.010 & 0.010 & 0.010 \\
\hline \multirow{3}{*}{ Dose Increase } & Before 2006 & 0.282 & 0.277 & 0.273 & 0.261 \\
\hline & 2006-2010 & 0.242 & 0.234 & 0.233 & 0.223 \\
\hline & After 2010 & 0.225 & 0.216 & 0.215 & 0.198 \\
\hline
\end{tabular}


Table 7: Prevalence of patients by Charlson Index (Health Status)(TI2)

\begin{tabular}{l|ccc|ccc|ccc|ccc}
\hline & \multicolumn{4}{|c}{ Never } & \multicolumn{4}{c}{ Compliers, D=0 } & \multicolumn{3}{c}{ Compliers, D=1 } & \multicolumn{3}{c}{ Always } \\
\hline Variable & Pre & Dur. & Post & Pre & Dur. & Post & Pre & Dur. & Post & Pre & Dur. & Post \\
\hline Charlson very low & 0.49 & 0.39 & 0.34 & 0.48 & 0.40 & 0.37 & 0.49 & 0.36 & 0.31 & 0.47 & 0.36 & 0.33 \\
Charlson low & 0.30 & 0.33 & 0.32 & 0.30 & 0.32 & 0.32 & 0.29 & 0.33 & 0.32 & 0.31 & 0.33 & 0.32 \\
Charlson med/high & 0.13 & 0.17 & 0.19 & 0.14 & 0.17 & 0.18 & 0.14 & 0.19 & 0.20 & 0.14 & 0.18 & 0.19 \\
Charlson high & 0.08 & 0.11 & 0.15 & 0.07 & 0.11 & 0.13 & 0.08 & 0.12 & 0.17 & 0.08 & 0.13 & 0.16 \\
\hline
\end{tabular}

Note: The groups in this table are defined as follows.

- "Never": Patient dose does not change during the year, among those patients seeing a physician with an above average propensity to switch dose among their other patients;

- "Compliers": Patient dose switches or does not switch during the year depending on whether the physician propensity to switch dose among other patients is above or below average;

- "Always": Patient switches dose during the year, among those patients seeing a physician with a below average propensity to switch dose among their other patients. 
Table 8: Distribution of Number of Liver Tests (TI3)

\begin{tabular}{l|l|l|l}
\hline Number & Overall & Lower dose & Higher dose \\
\hline \multicolumn{4}{|c}{ Before 2006} \\
\hline 1 & $0.079^{* * *}$ & $0.008^{* * *}$ & $0.075^{* * *}$ \\
2 & $0.087^{* * *}$ & $0.010^{* * *}$ & $0.078^{* * *}$ \\
3 & $0.087^{* * *}$ & $0.011^{* * *}$ & $0.078^{* * *}$ \\
4 & $0.143^{* * *}$ & $0.021^{* * *}$ & $0.135^{* * *}$ \\
5 & $0.167^{* * *}$ & $0.027^{* * *}$ & $0.162^{* * *}$ \\
\hline \multicolumn{4}{|c}{$2006-2010$} \\
\hline 1 & $0.083^{* * *}$ & $0.008^{* * *}$ & $0.080^{* * *}$ \\
2 & $0.087^{* * *}$ & $0.011^{* * *}$ & $0.083^{* * *}$ \\
3 & $0.081^{* * *}$ & $0.010^{* * *}$ & $0.077^{* * *}$ \\
4 & $0.137^{* * *}$ & $0.018^{* * *}$ & $0.131^{* * *}$ \\
5 & $0.136^{* * *}$ & $0.017^{* * *}$ & $0.130^{* * *}$ \\
\hline \multicolumn{4}{|c}{ After 2010} \\
\hline 1 & $0.067^{* * *}$ & $0.011^{* * *}$ & $0.061^{* * *}$ \\
2 & $0.081^{* * *}$ & $0.010^{* * *}$ & $0.077^{* * *}$ \\
3 & $0.063^{* * *}$ & $0.006^{* * *}$ & $0.060^{* * *}$ \\
5 & $0.104^{* * *}$ & $0.013^{* * *}$ & $0.099^{* * *}$ \\
5 & $0.094^{* * *}$ & $0.012^{* * *}$ & $0.088^{* * *}$ \\
\hline
\end{tabular}

Table 9: Patient Adherence Rate and Mean Serum Cholesterol (mmol/L) (TI5)

\begin{tabular}{l|ccc|ccc|ccc|ccc}
\hline & \multicolumn{4}{|c}{ Never } & \multicolumn{4}{c}{ Compliers, D=0 } & \multicolumn{3}{c}{ Compliers, D=1 } & \multicolumn{3}{c}{ Always } \\
\hline Variable & Pre & Dur. & Post & Pre & Dur. & Post & Pre & Dur. & Post & Pre & Dur. & Post \\
\hline Adherence & 0.54 & 0.66 & 0.69 & 0.53 & 0.66 & 0.68 & 0.62 & 0.74 & 0.74 & 0.61 & 0.74 & 0.75 \\
HDL chol. & 1.43 & 1.41 & 1.42 & 1.44 & 1.41 & 1.44 & 1.44 & 1.39 & 1.40 & 1.43 & 1.38 & 1.38 \\
LDL chol. & 3.29 & 3.04 & 2.84 & 3.33 & 3.06 & 2.85 & 3.54 & 3.22 & 3.12 & 3.54 & 3.24 & 3.10 \\
Tot. chol. & 5.53 & 5.19 & 4.95 & 5.57 & 5.22 & 4.99 & 5.85 & 5.42 & 5.26 & 5.85 & 5.46 & 5.26 \\
\hline
\end{tabular}

Note: The groups in this table are defined as follows.

- "Never": Patient dose does not change during the year, among those patients seeing a physician with an above average propensity to switch dose among their other patients;

- "Compliers": Patient dose switches or does not switch during the year depending on whether the physician propensity to switch dose among other patients is above or below average;

- "Always": Patient switches dose during the year, among those patients seeing a physician with a below average propensity to switch dose among their other patients. 
Figure 1: Physicians Prescribe Higher Equipotency Doses (TI4)

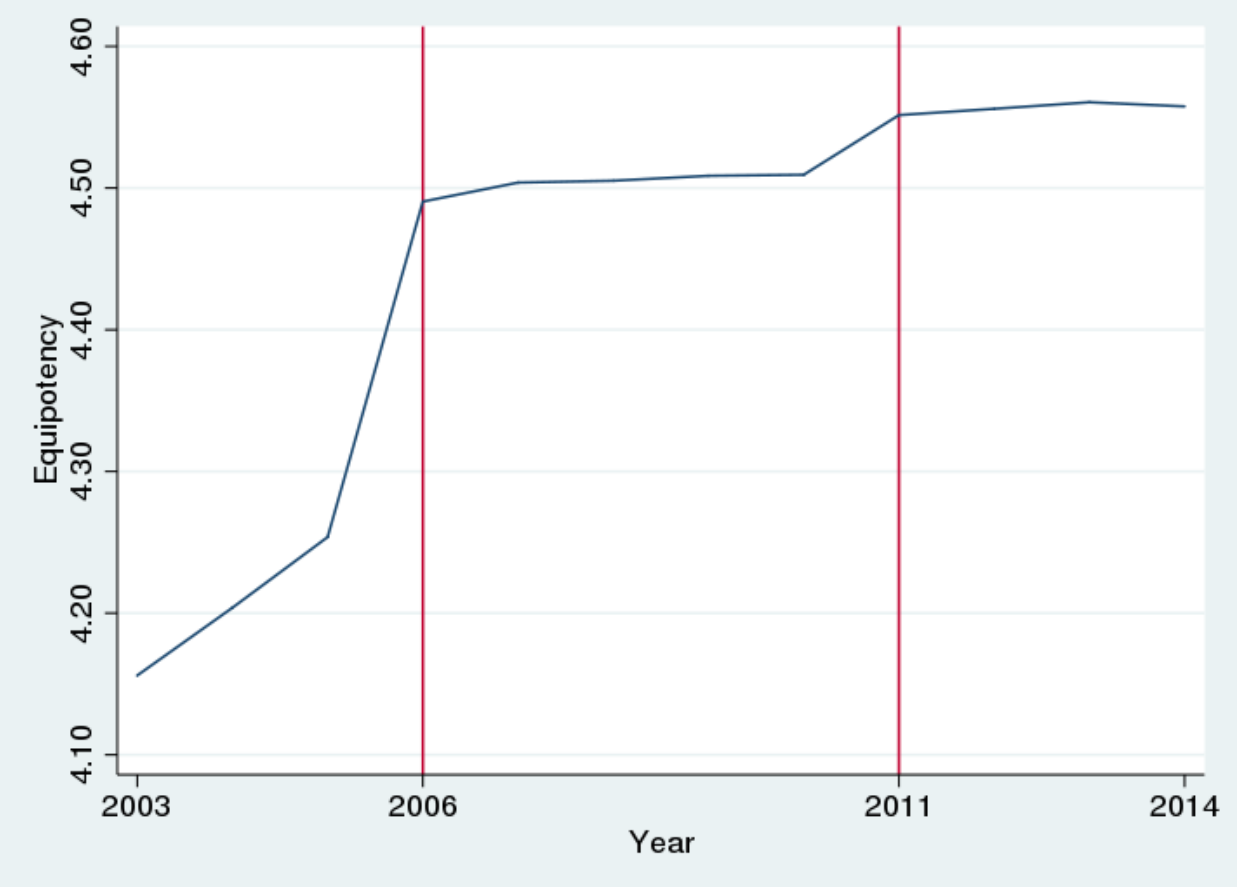


Figure 2: Physicians Prescribe Higher Equipotency Doses (by Charleson Score) (TI4)

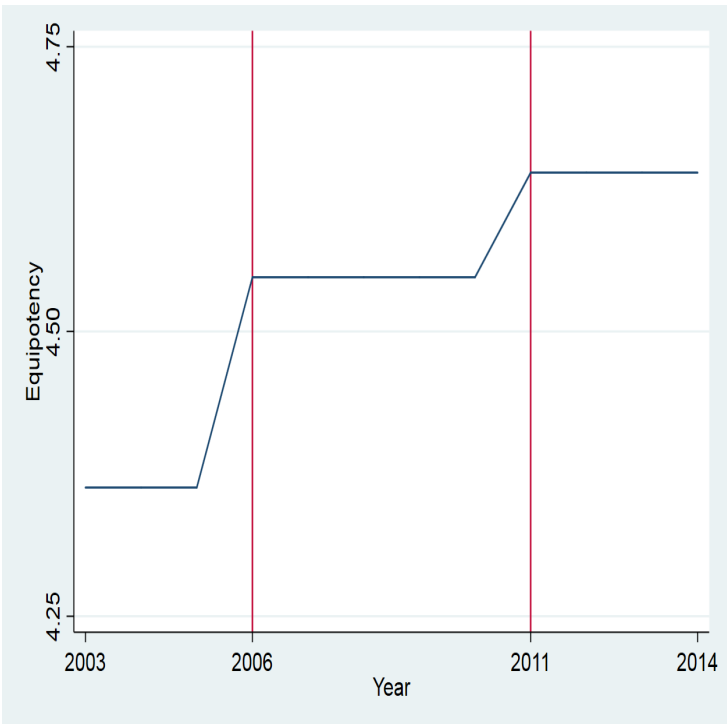

(a) Chalrson: 1

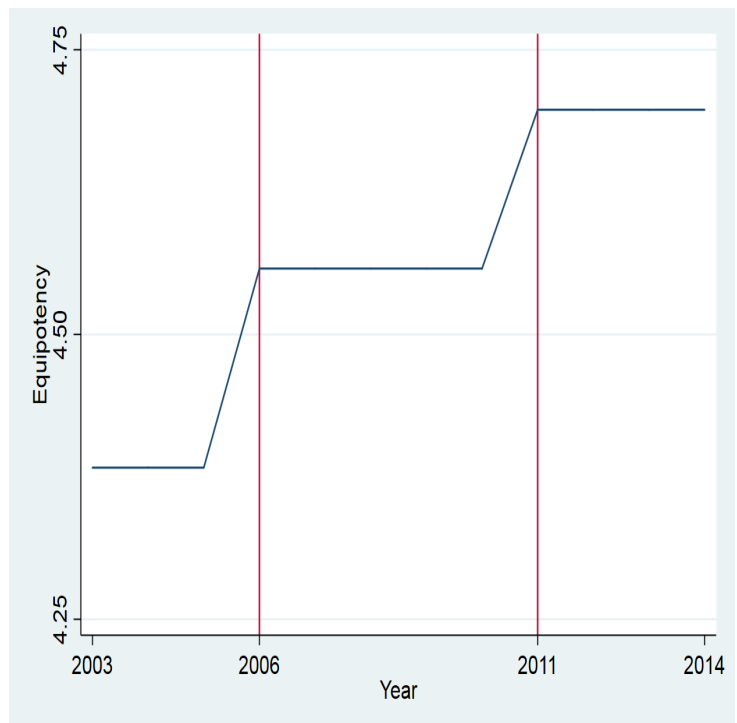

(c) Chalrson: 3

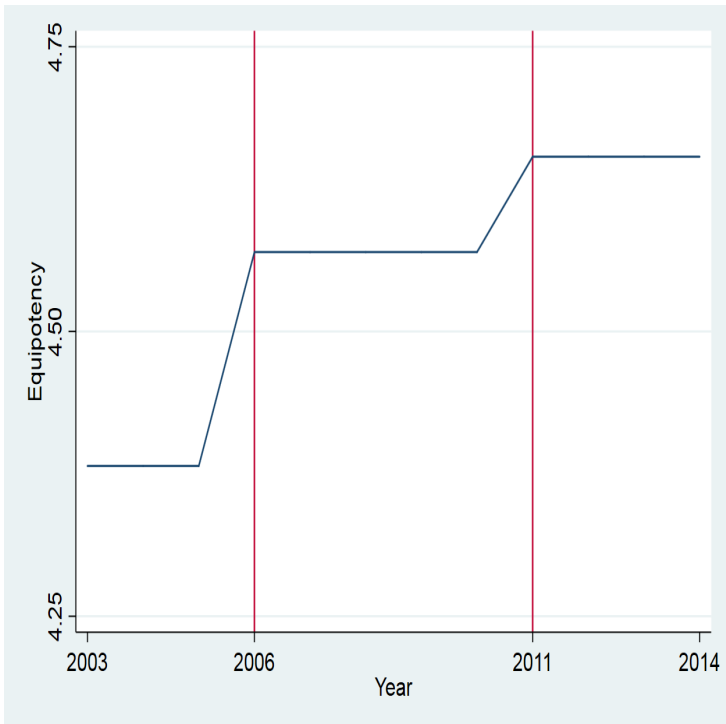

(b) Chalrson: 2

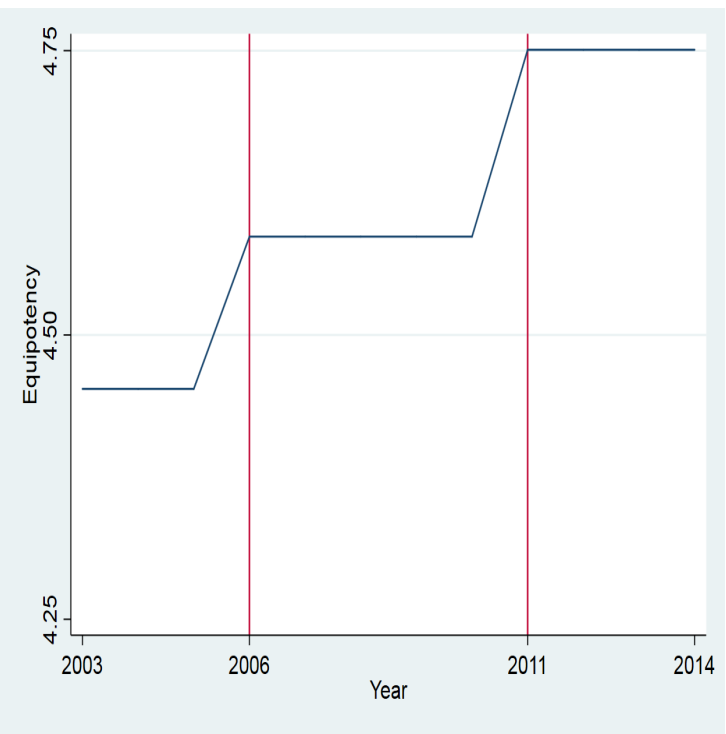

(d) Chalrson: $\geq 4$ 